\title{
The properties of the kinematically distinct components in NGC 448 and NGC 4365
}

\author{
B. Nedelchev ${ }^{1,2}$, L. Coccato ${ }^{2}$, E. M. Corsini ${ }^{3,4}$, M. Sarzi ${ }^{1,9}$, T. de Zeeuw ${ }^{2,5,6}$, A. Pizzella ${ }^{3,4}$, E. Dalla Bontà ${ }^{3,4}$, \\ E. Iodice ${ }^{7}$, and L. Morelli ${ }^{8}$
}

${ }^{1}$ Centre for Astrophysics Research, University of Hertfordshire, College Lane, Hatfield AL10 9AB, UK e-mail: b.nedelchev@herts.ac.uk

2 ESO, Karl Schwarzschild Strasse 2, 85748 Garching b. München, Germany

3 Dipartimento di Fisica e Astronomia "G. Galilei”, Università di Padova, vicolo dell'Osservatorio 3, 35122 Padova, Italy

4 Max-Planck-Institut für extraterrestrische Physik, Giessenbachstrasse, 85741 Garching, Germany

5 Sterrewacht Leiden, Leiden University, Postbus 9513, 2300 RA Leiden, The Netherlands

6 INAF-Osservatorio Astronomico di Capodimonte, via Moiariello 16, 80131 Napoli, Italy

7 INAF-Osservatorio Astronomico di Padova, vicolo dell'Osservatorio 5, 35122 Padova, Italy

8 Instituto de Astronomia y Ciencias Planetarias, Universidad de Atacama, Copiapò, Chile

9 Armagh Observatory and Planetarium, College Hill, Armagh BT61 9DG, UK

Received 16 February 2018 / Accepted 17 September 2018

\begin{abstract}
Aims. We study the kinematically distinct components in two early-type galaxies NGC 448 and NGC 4365 aided by integral-field observations with the Multi-Unit Spectroscopic Explorer (MUSE) on the Very Large Telescope. The former galaxy has previously been shown to host a counter-rotating stellar disc while the latter harbours a central (apparently) decoupled core that has been suggested to not be physically distinct from the main body and instead stems from the different orbital types in the core and main body due to its triaxial nature. We aim to measure the brightness profiles, kinematics, and stellar population properties of the peculiar kinematic structures in these galaxies and shed light on their true nature and formation mechanism.

Methods. We use a kinematic decomposition technique to separate the individual contributions to the spectra of the two distinct kinematic components observed at each spatial position in the field of view. Furthermore, by folding back the outcome of a photometric decomposition we reduce the intrinsic degeneracies in recovering the kinematics and the best-fitting stellar spectral templates. Finally, by extracting the Lick line-strength indices for the individual components and fitting them to single stellar population models we derive their ages, metallicities, and $\alpha / \mathrm{Fe}$ overabundances.

Results. The two kinematically decoupled stellar components in NGC 448 have similar ages, but different chemical compositions. The distinct kinematic feature in NGC 448 has a nearly exponential surface-brightness light profile, dominates in the innermost $\sim 10^{\prime \prime}$, is smaller in size, and is very likely an embedded counter-rotating disc as also indicated by its kinematics. It has higher metallicity than the main galaxy stellar body and lower $\alpha /$ Fe overabundance. By contrast, we do not find evidence for true decoupling in the two distinct kinematic components in NGC 4365. This confirms earlier work suggesting that the kinematically distinct core is likely not a separate dynamical structure, but most certainly likely a projection effect stemming from the orbital structure of this galaxy that was previously found to be intrinsically triaxial in shape.

Conclusions. Our findings indicate that the kinematically decoupled component in NGC 448 is truly decoupled, has external origin, and was formed through either the acquisition of gas and a subsequent star-formation episode or from the direct accretion of stars from a companion. Conversely, the presence of a kinematically distinct component in NGC 4365 is not associated to a true kinematic decoupling and is instead most likely due to a projection effect stemming from the triaxial nature of this galaxy.
\end{abstract}

Key words. galaxies: abundances - galaxies: kinematics and dynamics - galaxies: stellar content - galaxies: individual: NGC 448 galaxies: individual: NGC 4365 - galaxies: elliptical and lenticular, cD

\section{Introduction}

The kinematic and stellar population properties of local galaxies carry the imprint of their formation and evolution histories. Within the local Universe, most galaxies display rather regular kinematic appearance (Krajnović et al. 2008; Cappellari 2016). However, a fraction of the local galaxies show some peculiarities in their stellar (e.g. Krajnović et al. 2011) or gas (e.g. Sarzi et al. 2006) kinematics or both. Often these peculiarities imply under-

* This work is based on observations taken at ESO La Silla Parnal Observatory within the program 094.B-0225(A). lying rotation around more than one axis (or anti-parallel spin), characterising a class of galaxies labelled as "multi-spin galaxies" (Rubin 1994). The observed multiple-spin axes could arise from a number of morphologically different structures. These range from polar ring or disc structures (e.g. NGC 4650A, Iodice et al. 2015) and large-scale counter-rotating discs (e.g. NGC 4550, Coccato et al. 2013), to kinematically distinct or counter-rotating cores (e.g. NGC 5813, Krajnović et al. 2015). The latter population of galaxies with a kinematically distinct core (KDC) displays an abrupt change in the direction of the velocity field accompanied by a large change in the kinematic position angle. In some of these special cases the velocity of the KDC switches sign (i.e. the two 
kinematic components show opposite rotation to one another) to differentiate a subclass of KDC whose members are specifically defined as having counter-rotating cores (CRC). Another signature of a counter-rotating component is the presence of two symmetric velocity dispersion peaks along the main rotation axis in a subclass of multi-spin galaxies labelled as " $2 \sigma$ " (Krajnović et al. 2011). "Intrinsic" counter rotation is observed when the rotation occurs around the same rotation axis, but involves different kinematic components rotating in opposite directions. Conversely, if the (decoupled) components have different misaligned rotation axes and their angular momentum vectors simply project antiparallel onto the sky plane, the counter-rotation is deemed to be "apparent" (Corsini et al. 2014).

Observationally, a number of disc galaxies (i.e. some lenticulars and spirals) have been demonstrated to possess a truly decoupled kinematic structure that counter-rotates with respect to the main stellar body and often has a disc-like morphology (e.g. Coccato et al. 2011, 2013, 2015; Katkov et al. 2013; Morelli et al. 2017). Either using long-slit or integral-field unit (IFU) spectroscopic observations, in conjunction with a spectroscopic decomposition technique (Coccato et al. 2011), has revealed that the distinct cores of such galaxies are more extended embedded components, comprise younger stellar populations, and are always accompanied by a substantial gas reservoir traced by ionised gas emission (Johnston et al. 2013; Pizzella et al. 2014; Mitzkus et al. 2017).

Owing to their peculiar nature, galaxies with counter-rotating components have been investigated with the aid of numerical simulations. Several different formation scenarios have been proposed and modelled to explain their existence. The most favoured formation channel for such kinematically distinct components in lenticulars and spirals is the external acquisition of gas as a consequence of a gas-rich major or minor merger and a subsequent period of in situ star formation (Pizzella et al. 2004). Detailed numerical modelling has indeed shown that both gaseous and stellar counter rotation can result from a minormerger scenario, provided that initially the two progenitors have opposite spins and that the gas content of one of the progenitors is substantially higher than that of the other such that the gas accreted on retrograde orbits is not dissipated and swept up by any pre-existing gas (e.g. Bassett et al. 2017). Another channel for the formation of disc-like counter-rotating distinct kinematic components is proposed in Algorry et al. (2014). The consequential gas accretion from two distinct large-scale cosmological filaments under a very specific spatial configuration in their zoom-in cosmological simulation naturally forms a population of counter-rotating stars implanted within the host galaxy. Evans \& Collett (1994) also suggested that counter-rotating stellar discs can form through the internal separatrix crossing mechanism. The change from elliptical to circular disc (e.g. through the disbandment of a bar structure in a triaxial halo) shifts some stars, initially moving on box orbits across the separatrix to tube orbits, shaping two co-rotating and counter-rotating stellar populations. Another mechanism that was put forward to explain the formation of two counter-rotating discs is the (near) coplanar major merger of two opposite spin spirals (e.g. Puerari \& Pfenniger 2001; Crocker et al. 2009). In such a merger the resultant prograde rotating stellar disc becomes "heated" more than the retrograde one to naturally explain the kinematic structure of galaxies similar to NGC 4550 (Crocker et al. 2009).

The situation is even more complicated with regards to ellipticals. Kinematically distinct cores have long been known to occur frequently in the centres of elliptical galaxies (e.g. Bender 1988). Generally, distinct kinematic features can be the tell-tale signs for either intrinsically more centrally concentrated embedded small components, or just "tip-of-the-iceberg" indication of more extended kinematic structures. Nevertheless, McDermid et al. (2006) reported a dichotomy in the KDCs found within massive ellipticals. The elliptical slow rotators mainly host more extended KDCs (on kiloparsec scales) with stellar populations with ages comparable to that of the stars within the main galaxy body. Conversely, the more centrally concentrated KDCs (of a few hundred parsecs) usually have noticeably younger stellar populations and are predominantly found in fast-rotating galaxies. The KDCs in massive ellipticals could also be a mere projection effect. The (weak) triaxial nature of some of these galaxies (Cappellari et al. 2007) allows them to support multiple types of different orbit families (e.g. de Zeeuw \& Franx 1991) and therefore viewed from a different vantage point these can appear to possess a KDC, nicely illustrated in Statler (1991).

A lot of effort from a simulation standpoint has also been focused towards understanding the nature of the kinematically distinct components within more massive ellipticals. Ever since their first detection these KDCs have been suggested to be the signature of a past merger (e.g. Kormendy 1984; Balcells \& Quinn 1990). Indeed, Hoffman et al. (2010) proposed that a merger event involving progenitors harbouring a non-negligible gas reservoir $(\sim 20 \%)$ can frequently result in elliptical galaxies that exhibit a KDC whose properties resemble that of a faster(counter-)rotating embedded disc. Bois et al. (2011) performed another comprehensive set of high-resolution merger simulations with various initial progenitor mass ratios. Their results showed that the remnants of a merger between two spiral galaxies with opposite spin would resemble slow rotators. Furthermore, the remnants would often harbour a central KDC, although these simulations did not manage to reproduce all the observed morphological and kinematic properties of the massive elliptical galaxies part of the ATLAS ${ }^{3 \mathrm{D}}$ survey (Cappellari et al. 2011).

Moreover, KDCs need not be just a consequence of a merger of opposite spin galaxies. The reactive force stemming from the substantial mass loss in the merger process can act to naturally place gas or stars, or both, on retrograde orbits and conspire to form a KDC embedded in the remnant elliptical galaxy (Tsatsi et al. 2015). Most recently, Schulze et al. (2017) followed the formation and evolution of a small-scale (and almost certainly therefore younger) KDC in a high-resolution equal-mass-merger simulation with specific emphasis on the KDC stability. Perhaps surprisingly, they discovered that such structures could be semistable for about $3 \mathrm{Gyr}$ and highly dynamic, undergoing global gyroscopic precession, before being gradually dissolved and dispersed within a few gigayears.

In this paper, we study the kinematically distinct components in NGC 448 and NGC 4365 with the aim to disentangle their kinematics, stellar population properties, and morphologies. These early-type galaxies were selected from the ATLAS ${ }^{3 \mathrm{D}}$ survey as part of an effort to address the nature of kinematically distinct components. They were chosen to be the hosts of a pronounced kinematically distinct component. The visible apparent size (diameter) of the kinematical peculiarities in previous observations of these two galaxies was selected to be more than $10^{\prime \prime}$ to further safeguard against potential complications that can arise whilst trying to apply our kinematic decomposition technique and ensure its robust performance. Katkov et al. (2016) independently studied NGC 448 and extracted a long-slit spectrum along the galaxy major axis to perform a thorough investigation of the properties of the two kinematically decoupled components. Our second galaxy NGC 4365 was also extensively observed with the SAURON integral-field 
spectrograph (Davies et al. 2001) and comprehensive dynamical modelling by means of the Schwarzschild (1979) orbit superposition method has shown that the KDC is not necessarily a true kinematically decoupled structure, but instead appears to be an artefact due to the triaxial nature of NGC 4365 (van den Bosch et al. 2008).

This paper is organised as follows. In Sect. 2 we describe the observations and detail the performed data reduction. Section 3 outlines our photometric analysis of the two galaxies. In Sect. 4 we describe the method by which we obtained the onecomponent kinematics and the resultant kinematics for both galaxies. Then we provide a short summary of the kinematic decomposition technique and present the outcome of such a decomposition. Section 5 presents the results of our measurements of the strength of the absorption lines as well as the results of our single-stellar-population modelling for the individual kinematic components of NGC 448. Finally, in Sect. 6 we discuss our finding in light of the possible formation scenarios of the KDCs in these galaxies and summarise our conclusions.

\section{Observations and data reduction}

The spectroscopic observations were carried out with the MUSE integral field spectrograph mounted on the UT4 telescope of the Very Large Telescope at the Paranal Observatory (Chile). Observations were taken in November and December, 2014 (NGC 448), and on 12 February, 2015 (NGC 4365), in service mode during grey time and photometric conditions. The seeing was about $1^{\prime \prime}$, as measured from the ESO meteo monitor. MUSE was configured in wide-field mode, without adaptive optics, and in nominal-wavelength mode; this setup ensured a field of view of $1^{\prime} \times 1^{\prime}$, a spatial sampling of $0 . ' 2 \times 0 .{ }^{\prime} 2$ per pixel, a spectral coverage of 4800-9300 $\AA$, and a spectral sampling of $1.25 \AA$ pixel $^{-1}$.

The observations were organised in several exposures of $1260 \mathrm{~s}$ intervals with offset sky exposures of $270 \mathrm{~s}$ in between. Scientific exposures were dithered by $\sim 1^{\prime \prime}$ and rotated by $90^{\circ}$ with respect to each other to minimise the instrumental signature of the different spectrograph on the combined data cubes. The total exposure times are $42 \mathrm{~m}$ and $2.9 \mathrm{~h}$ for NGC 4365 and NGC 448, respectively.

The basic data reduction (bias subtraction, flat fielding, and wavelength calibration) was carried out using the MUSE ESO pipeline version 1.6.2 (Weilbacher et al. 2012). We also utilised the Zurich Atmosphere Purge (ZAP) sky subtraction procedure (Soto et al. 2016) to remove any residual sky features left over by the main MUSE ESO data-reduction pipeline. The ZAP code implements a sky-subtraction method based on a principal component analysis complemented with data filtering and segmentation that allows robust flux preservation with negligible or no impact on any astronomical source line profiles. These two procedures were executed through their ESO Reflex environment (Freudling et al. 2013) implementations. In the case of NGC 448, the ZAP cleaning was done by directly evaluating the sky on the object cube itself. For NGC 4365, we used the dedicated sky exposures as the galaxy occupied the whole field of view, therefore leaving no spaxels dominated purely by the sky signal. As a subsequent step we ran the Cappellari \& Copin (2003) Voronoi binning algorithm aiming to achieve a signal-to-noise ratio $(\mathrm{S} / \mathrm{N})$ of 90, evaluated considering the whole MUSE spectral wavelength range. We consider the mean of the spectrum as the signal, and the noise as the square root of the mean of the variance returned by the MUSE ESO pipeline. Furthermore, to suppress any of the low-signal levels in the outer parts of the observed field of NGC 448 we chose to apply a further elliptical mask aligned to the galaxy position angle (PA) with a semi-major to semi-minor axis ratio of 1.5 on the MUSE reconstructed whitelight image with a semi-major axis of 190 spaxels, thereby minimising the effect of the outer regions with very low $\mathrm{S} / \mathrm{N}$ on the size of the bins in sections of higher $\mathrm{S} / \mathrm{N}$. No additional masking was used in the case of NGC 4365.

\section{Photometric decomposition}

In this section we exploit the exquisite imaging capabilities of MUSE by analysing the galaxy images obtained by collapsing the datacubes along wavelength. The purpose is to identify the presence of photometrically distinct components and compare their properties with those of the structural components that we would identify via the spectroscopic decomposition (see Sect. 4.3). In this way it is possible to i) establish a direct association between photometric and kinematic components; and ii) to use the photometric information as priors in the spectroscopic decomposition to constrain the flux of the components. The use of photometric priors in the spectroscopic decomposition to improve the accuracy of the kinematics and stellar population measurements has been successfully applied in several cases (e.g. Coccato et al. 2014, 2015; Sarzi et al. 2016; Tabor et al. 2017).

The photometric decomposition is performed using the twodimensional (2D) fitting algorithm galfit (Peng et al. 2002). We use the reconstructed images rather than independent archive images in order to have the same conditions (e.g. seeing, sky background contamination) as the spectroscopic data. Indeed, the purpose is not to retrieve accurate parameters of the structural components (e.g. seeing-corrected scale radii), but to obtain good fitting surface brightness profiles that contain the same observational effects as the spectra, in order to eventually use them as constraints in the spectral decomposition.

In our decomposition, all components are parametrised with Sérsic profiles, which allows for the behaviour of both disc and spheroidal structures to be encapsulated. Our fits include only the minimum number of components required to reach a good fit, rejecting additional components that would contribute only a relatively small fraction of the stellar light (e.g. less than the $5 \%$ freedom that we already allow in our spectroscopic decomposition when adopting photometric priors, Sect. 5.1). For NGC 448, we identified three Sérsic components and for NGC 4365 we identified two. We also note that fits based on one Sérsic component never lead to satisfactory models for the observed surface-brightness distribution, in particular in the regions where we observe a KDC signature. Table 1 summarises the properties of our identified components, whereas in Fig. 1 we compare their surface brightness radial profiles with those measured on the reconstructed images using the iraf task ellipse (Jedrzejewski 1987).

As regards NGC 448, we already report here that the sum of the first two photometric components in NGC 448 is likely associated with the kinematic counter-rotating component, whereas the third photometric component is in fact ascribed to the main galactic stellar kinematic component. We also note that within the MUSE field of view, our photometric model agrees well with what was predicted by Katkov et al. (2016) on the basis of their innermost first three components, consisting of a Sérsic and two exponential profiles. Indeed, even though their fourth and most extended Sérsic component is responsible for $17 \%$ of the total light of NGC 448, this halo component does not contribute much 
Table 1. Photometric components in NGC 448 and NGC 4365.

\begin{tabular}{cccccc}
\hline \hline Name & mag & $R_{\mathrm{e}}$ & $\mathrm{n}$ & $\mathrm{b} / \mathrm{a}$ & $\mathrm{PA}$ \\
\hline KDC (NGC 448) & 19.32 & 1.04 & 1.16 & 0.89 & -52.9 \\
KDC (NGC 448) & 17.25 & 6.50 & 1.03 & 0.28 & -61.0 \\
Main (NGC 448) & 16.11 & 22.40 & 1.89 & 0.89 & -65.0 \\
KDC (NGC 4365) & 6.70 & 27.72 & 1.46 & 0.77 & 44.3 \\
Main (NGC 4365) & 9.10 & 3.90 & 0.98 & 0.75 & 42.7 \\
\hline
\end{tabular}

Notes. The first column gives the description of the fitted photometric component. Second column is the integrated magnitude of the photometric component returned by galfit (see Sect. 3). The third column provides the estimated effective radius in arcsec. The fourth column outlines the best-fitting Sérsic index. The fifth column is the semi-minor to semi-major axial ratio of the best-fitting model. The last column is the position angle of the best-fitting Sérsic profile.

light within the MUSE field of view, dropping already to less than a $6 \%$ fractional contribution at a radius of $20^{\prime \prime}$.

As for NGC 4365, we note that although a single core-Sérsic profile can match well the light profile in the KDC region of this galaxy (Ferrarese et al. 2006), here we performed a twocomponent decomposition for the purpose of testing whether these would then match the results of a spectral decomposition. As discussed below, we find no evidence for two structurally different stellar components in NGC 4365.

\section{Stellar kinematics}

\subsection{One-component kinematic fit}

As a first step in extracting the individual properties of our two distinct stellar structural components in these galaxies we used the Cappellari \& Emsellem (2004) Penalized Pixel-Fitting (pPXF) and the Gas AND emission Line Fitting (GANDALF) Sarzi et al. (2006) methods to recover the velocity, velocity dispersion, and the h3 and h4 coefficients of the Gauss-Hermite parametrisation van der Marel \& Franx (1993) of the line-ofsight (stellar) velocity distribution (LOSVD) and any potential ionized-gas emission line properties in each Voronoi bin. The pPXF procedure uses a model that is parametrised in terms of Gauss-Hermite functions for the LOSVD, and a set of linearly combined template spectra to best match the observed galaxy spectrum in pixel space. An important requirement for the extraction of an accurate LOSVD is that the spectral resolution of the templates provided to build the observed galaxy spectrum matches the instrumental one. The spectral resolution was measured by fitting a high-resolution solar spectrum template to the twilight spectra, after having combined them following the same sequence of the science observations, similar to (Sarzi et al. 2018). The measured instrumental FWHM is $2.8 \AA$, its variation across the field of view and the wavelength range is $\sim 0.1 \AA$. The adopted method ensures the measurement of the effective instrumental spectral resolution, which includes both the instrument properties and data reduction steps. With these considerations in mind we adopted as template spectra the Vazdekis et al. (2012, MIUSCAT) stellar population synthesis models (SSPs), spanning the broad 3465-9469 wavelength range based on the Girardi et al. (2000) stellar isochrones with $-0.71<[\mathrm{Z} / \mathrm{H}]<+0.22,0.06<t<18 \mathrm{Gyr}$, and with a unimodal initial-mass function with a power-law slope coefficient of 1.3 (Salpeter 1955). This stellar population synthesis model template library with a constant FWHM
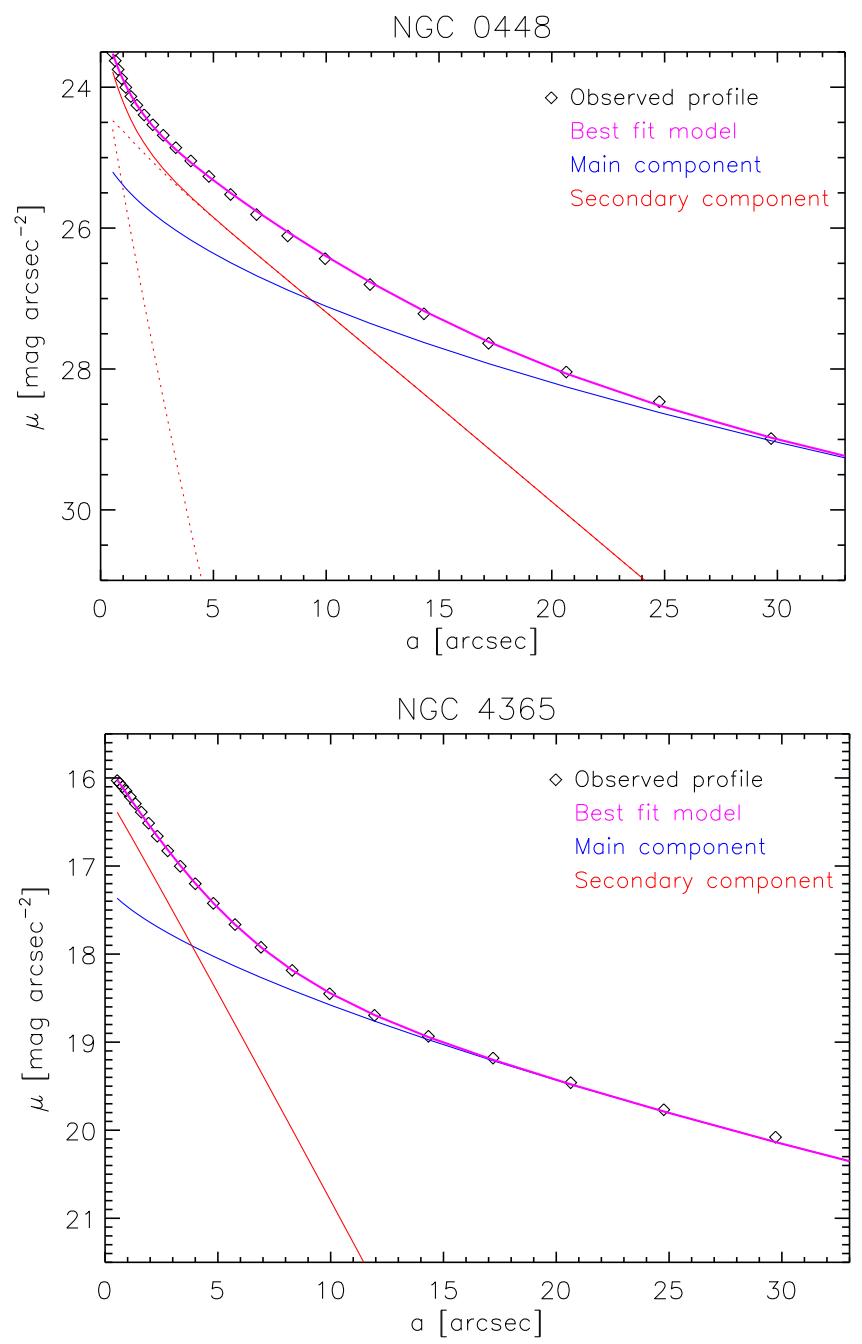

Fig. 1. Photometric decomposition for NGC 448 (upper panel) and NGC 4365 (lower panel). Each plot shows: the surface brightness profile measured from the reconstructed images (diamonds), the best fit model (magenta) as obtained from the combination of the structural components that are associated to the main galaxy (blue) and to the kinematically distinct core (KDC, labelled as "secondary component", in red). In the case of NGC 448, the KDC is assumed to include two subcomponents (dashed red lines).

resolution of $2.8 \AA$ was retrieved through the on-line portal $^{1}$. Prior to performing both pPXF and GANDALF fits, the spectra of both our template library and observations were logarithmically re-binned to a common velocity step of $55.17 \mathrm{~km} \mathrm{~s}^{-1}$. Due to the higher spectral resolution and relatively high $\mathrm{S} / \mathrm{N}$ of the $\mathrm{CaT}$ absorption lines we extracted the kinematics of NGC 448 within the 8350-8900 $\AA$ spectral region. A first degree additive polynomial and a thirddegree multiplicative polynomial were used to account for template mismatch, any imperfections in the sky subtraction procedure, and potential inaccuracies stemming from the spectral calibration process. For NGC 4365, we instead opted to extract the kinematics by creating an optimal template to match the summed up spectrum within a central circular aperture by performing an initial pPXF fit. This template and a high-order additive and multiplicative polynomial (15th and 15 th degree, respectively) were later adopted to similarly extract the

\footnotetext{
1 http://www.iac.es/proyecto/miles/pages/webtools/ tune-ssp-models.php
} 

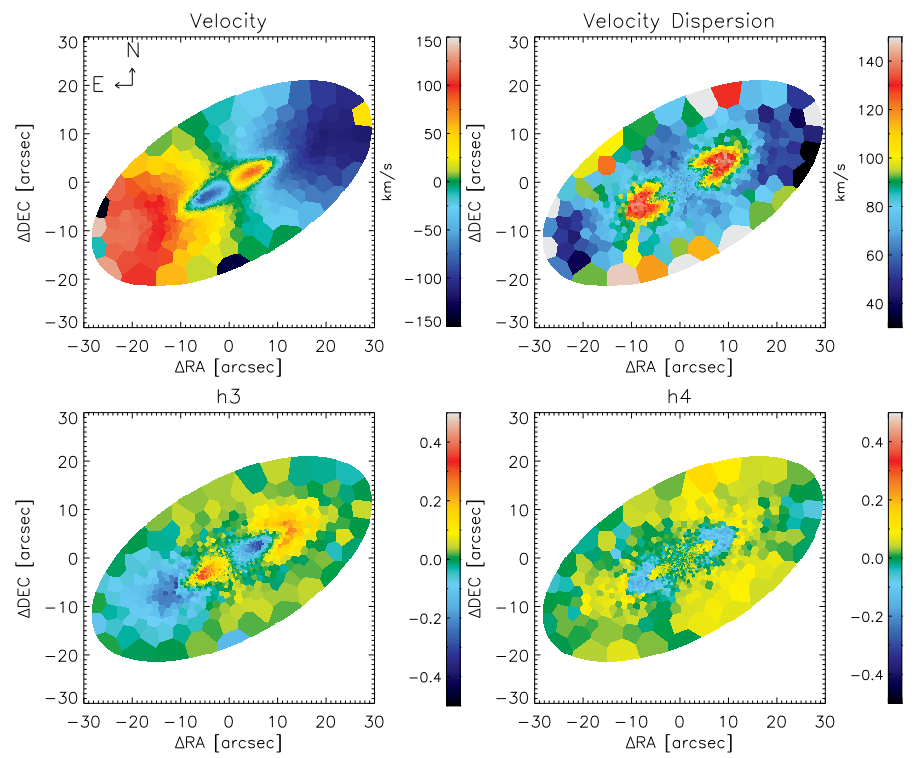

Fig. 2. The one-component kinematic maps for NGC 448. Top left: retrieved velocity field. Top right: velocity dispersion field. Bottom left: map of the h3 Gauss-Hermite coefficient in the parametrisation of the LOSVD. Bottom right: map of the h4 Gauss-Hermite coefficient in the parametrisation of the LOSVD.

one-kinematic-component maps through pPXF and GANDALF within each Voronoi bin.

Figure 2 presents the 2D kinematic maps for NGC 448 extracted by performing the procedure described above. We observe abrupt changes in the direction of the velocity field (upper-left panel) with a maximum rotation amplitude of $\sim 140 \mathrm{~km} \mathrm{~s}^{-1}$. There is a pronounced anti-correlation between the velocity and $\mathrm{h} 3$ coefficient in the outer regions of the galaxy, plausibly due to the presence of an extended disc component that rotates in a noticeably different manner to that of the main stellar body. Moreover, within the inner central part, this anticorrelation persists even after the velocity field switches direction. We confirm the classification of NGC 448 as a " $2 \sigma$ " galaxy (top-right panel), because of the two pronounced symmetric offcentre peaks of $\sim 230 \mathrm{~km} \mathrm{~s}^{-1}$ in the velocity dispersion map. The behaviour of the h4 Gauss-Hermite coefficient used to capture symmetric departures from purely Gaussian LOSVD profile indicates that at the positions where the velocity dispersion is at its highest values the LOSVD has pronounced, extended "wings". Taken together, such kinematic behaviour in NGC 448 is found to be symptomatic of the presence of an embedded central intrinsically counter-rotating disc (Rubin et al. 1992; Rix et al. 1992; Bertola et al. 1996; Cappellari et al. 2007; Vergani et al. 2007) that from these maps appears to span at least $~ 13^{\prime \prime}$ in its radial extent.

In Fig. 3 we show the 2D kinematic maps for NGC 4365. The velocity field (upper left panel) displays a clear "four-part counter-rotating" morphology because it possesses four maxima at different amplitudes and positions and the velocity field has a distinctive "S-shape" twist (Statler 1991). The central velocity extrema have an amplitude of $\sim 80 \mathrm{~km} \mathrm{~s}^{-1}$, whereas in the outer regions the velocity amplitude at the extrema is smaller $\left(\sim 55 \mathrm{~km} \mathrm{~s}^{-1}\right)$. The velocity dispersion (upper-right panel) is centrally peaked with a maximum value of $\sim 270 \mathrm{~km} \mathrm{~s}^{-1}$. The h3 coefficient (bottom-left panel) strongly correlates with the observed velocity pattern where we also observe a kinematically distinct behaviour for the velocity field. Such correlation is less pronounced in the outer parts.
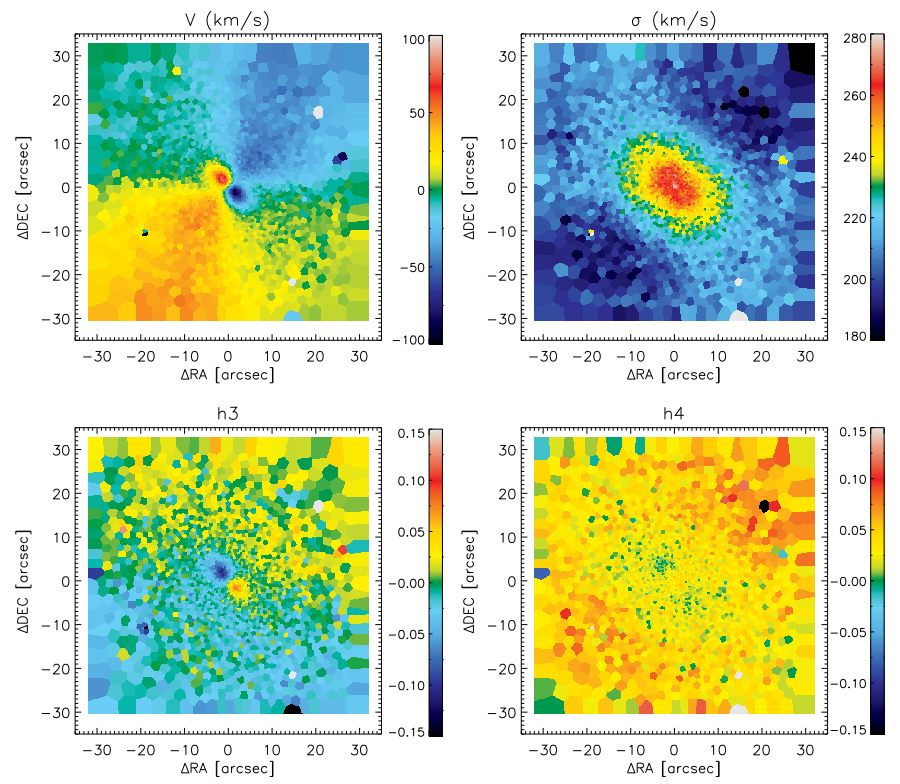

Fig. 3. One-component kinematic maps for NGC 4365. Top left: retrieved velocity field. Top right: velocity dispersion field. Bottom left: map of the h3 Gauss-Hermite coefficient in the parametrisation of the LOSVD. Bottom right: map of the h4 Gauss-Hermite coefficient in the parametrisation of the LOSVD.

\subsection{The kinematic decomposition procedure}

To derive the individual properties of the two different structural components, we followed a procedure identical to that of Coccato et al. (2011). This so-called kinematic decomposition method takes advantage of the characteristic spectral imprint (e.g. asymmetric or double trough line profiles) of a spectrum, which constitutes two overlapping stellar populations of different kinematics. The method builds on pPXF in that it aims to model the observed spectra and therefore to also achieve a separation of two possible stellar kinematic structural components in every spatial bin, by constructing a unique set of linearly combined templates for each component convolving it with a separate Gaussian line-of-sight velocity distribution function. To account for any potential inaccuracies in the spectral calibration and the effects induced by the reddening from dust on the spectra similarly to $\mathrm{pPXF}$, our procedure adopts multiplicative Legendre polynomials of a preselected order assumed to be identical for both components. We avoid the use of additive Legendre polynomials, because they could profoundly bias our line-strength measurements (Cappellari 2017). Given the complexity of this fitting approach, a useful simplification is achieved through the normalisation of both the galaxy spectra and templates to unity. In this way we are able to obtain the fractional contribution to the flux within a given wavelength range of both components in terms of a single parameter instead of assigning two separate light fraction contribution parameters. Due to the surprisingly good calibration in both our chosen set of templates and MUSE spectra, we found that such an arbitrary choice of normalising wavelength range does not influence any of the forthcoming conclusions and chose for simplicity to normalise all templates and MUSE spectra with respect to their mean levels. The main workhorse minimisation procedure within both PPXF and our kinematic decomposition method is the interactive data language (IDL) implementation (Markwardt et al. 2009) of the Levenberg-Marquardt least-squares curve-fitting algorithm that 


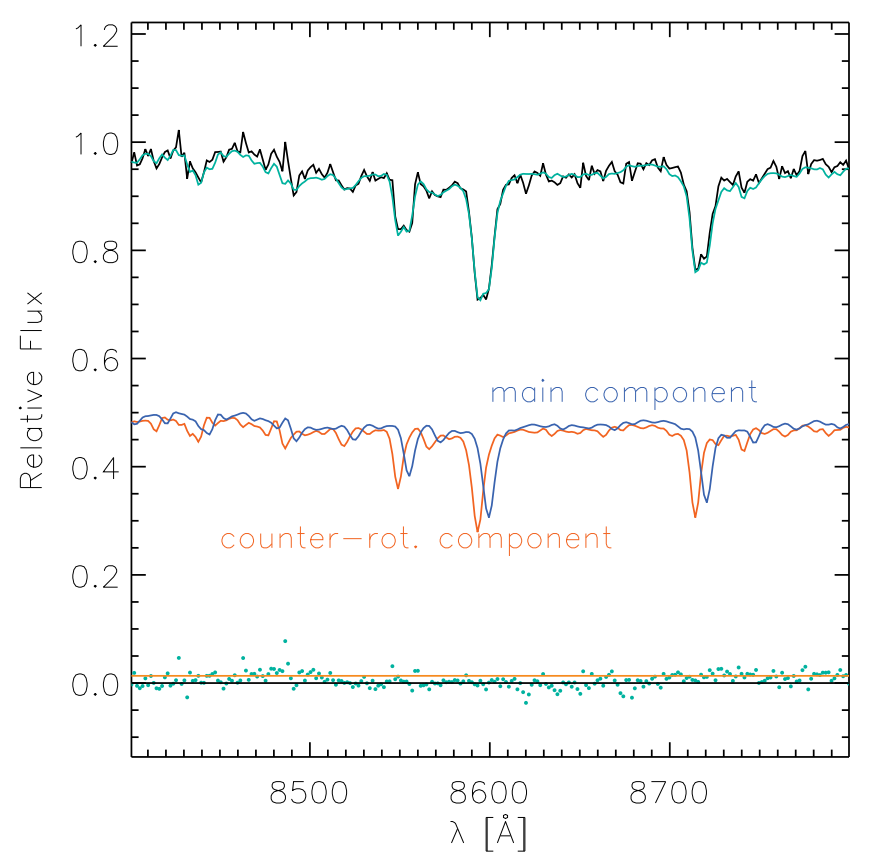

Fig. 4. Fit to the CaT spectral region for a bin of approximately 9 arcsec roughly along the major axis of the centre of the NGC 448 field where the two distinct kinematic components have an absolute velocity difference of $\sim 212 \mathrm{~km} \mathrm{~s}^{-1}$. The best-fitting model is in green. The contributions of the counter-rotating and main galaxy component are shown in blue and red, respectively. The green points signify the residual from our fitting and the orange line is set at the level of the resistant dispersion of the residual.

is sometimes found to converge, not necessarily towards a global minimum. We experimented with switching it with the slower, but potentially more sturdy, downhill simplex (AMOEBA) method (Nelder \& Mead 1965) with little to no success in line with the Cappellari (2017) finding that the minimisation algorithm does not noticeably influence the inferred pPXF results. Even though our fitting procedure is robust enough, it is not necessarily immune to completely unreasonable sets of initial guesses for the velocities of the two kinematic components. Therefore, we tested the impact of varying the initial guesses for both kinematic components (i.e. their velocities and velocity dispersions) in our decomposition model until we were convinced of the general validity of the results obtained.

\subsection{Two-component fit}

As described above, such a kinematic decomposition procedure allows us to simultaneously and independently retrieve the properties of the kinematic components, namely their velocity, velocity dispersion, fractional contribution to the flux (light fraction), and best-fitting stellar population template. Here, we focus on the individual photometric and kinematic properties of the observed distinct components of our galaxies.

\subsubsection{NGC 448}

Figure 4 shows an example spectrum in the CaT region for NGC 448 near the position of maximum velocity difference, where both components contribute almost the same fraction to the overall light (at $\sim 9^{\prime \prime}$ ). In the case of NGC 448 , the line profiles are clearly double peaked, highlighting the complex nature of the underlying stellar LOSVD. Figure 5 shows the reconstructed brightness maps averaged over the number of spaxels of both kinematic components. The light profiles taken along the photomet- ric major axis show that our kinematic and photometric decompositions agree remarkably well with each other (right panel of Fig. 5), so that the more (primary) and less (secondary) extended photometric components can be confidently attributed to the main and counter-rotating kinematic components, respectively. In this respect, we presently assume that the innermost photometric component belongs to the counter-rotating structure, consistent also with the conclusions of Katkov et al. (2016), although our data do not allow us to rule out that more central light could be assigned also to the main component. This limitation, however, does not affect our main conclusions. Further adaptive-opticsassisted MUSE observations may help in understanding the kinematics of NGC 448 in the very central few arcseconds.

We chose to discard all bins where either the fitting routine did not converge or the fractional light contribution of the counter-rotating component was less than $15 \%$. This somewhat arbitrary choice is motivated by the observation that the light of the less-extended kinematic component experiences a sharp rise at a radius of $\sim 19^{\prime \prime}$ along the pseudo-slit extracted from the IFU data, where its contribution to the total flux indeed drops below 0.15 . Furthermore, we retrieved the amplitude of the strongest CaT line and compared it to the level of the residual noise evaluated by computing the robust standard deviation of the residual (Beers et al. 1990, their Eq. (9)). The turn-off where the amplitude of the line drops below three times the residual noise level coincides roughly with our adopted light fraction threshold.

Nevertheless, because we did not impose any prior on the spatial extent of both of our kinematic components, the decomposition within the regions of small or zero velocity difference in between them erroneously detected a higher light fraction in one of the components. Figure 6 shows the 2D kinematic maps for NGC 448. The upper-left panel gives the retrieved velocity for the counter-rotating kinematic component. Both kinematic components rotate with comparable velocities and a maximum velocity amplitude for the counter-rotating component of $\sim 120 \mathrm{~km} \mathrm{~s}^{-1}$ at a distance of $\sim 11^{\prime \prime}$ from the centre of the galaxy. Its velocity dispersion is significantly lower (mean $\sigma_{\star}$ of $\sim 40 \mathrm{~km} \mathrm{~s}^{-1}$ ) in comparison to that of the main stellar body $\left(\sim 60 \mathrm{~km} \mathrm{~s}^{-1}\right)$, strengthening the idea that the counter-rotating component is indeed a disc. Driven by the excellent correspondence between the light profiles of the photometric components determined in Sect. 3 and those of the kinematic components, we decided also to impose the light contribution we obtained from photometry into our kinematic spectral model allowing only a small adjustment of at most 5\%. In Fig. 7 we show again the reconstructed surface brightness maps of both components and correspondingly their major-axis profiles. We consider again, as for our previous estimate, that we can reliably detect the counterrotating component when its contribution to the total flux is higher than 0.15. In the bins where this condition was met (left panel of Fig. 7) the mean absolute difference between the fixed photometric fractional contribution and the one retrieved by our previous analysis is $\sim 7.5 \%$. This difference is higher predominantly within the very central region, where the velocity difference between both kinematic components is rather small and thereby most likely did not originally allow a very robust kinematic decomposition. Even though fixing the photometric contributions is not strictly necessary, because of the complex nature of our kinematic decomposition method it relieves some of the parameter degeneracies. This, in turn, results in a more consistent parameter estimation. Moreover, in doing so we need not impose somewhat fiducial criteria to separate the Voronoi bins which contain both kinematic components from the ones where just a single one is present. Instead, as such a criteria, 

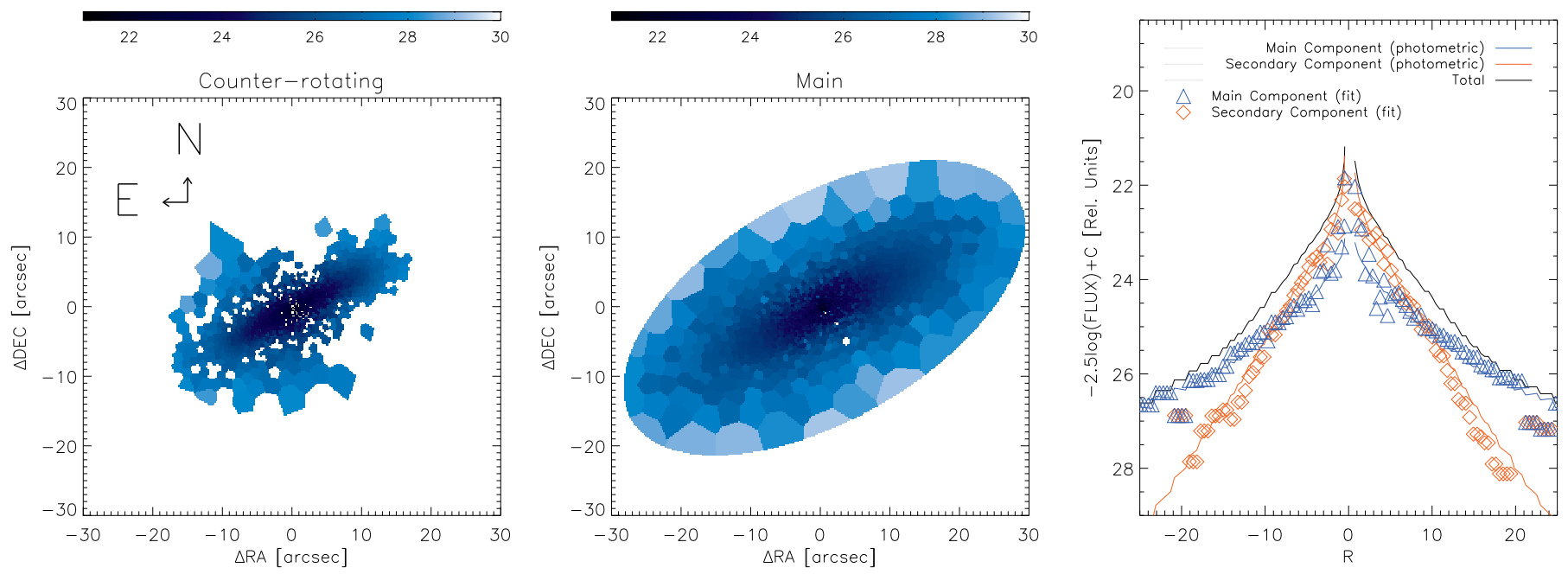

Fig. 5. Spectral flux maps and profile averaged over the number of spaxels for NGC 448. Left panel: reconstructed surface-brightness map of the counter-rotating component. Middle panel: reconstructed surface brightness map of the main component. Right panel: radial profile for both kinematic components, taken through a pseudo slit approximately aligned with the semi-major axis. The black, orange, and blue solid lines illustrate the photometrically derived total, the counter-rotating, and the main component flux contributions, respectively. The blue triangles and the orange diamonds show the flux contribution derived for the counter-rotating and main components, respectively, as derived through our fitting procedure without imposing any constraints.

we simply adopted the light fraction above which we deem the detection of the counter-rotating component reliable.

We now describe the kinematics obtained by imposing the photometric decomposition, which we adopt in the remainder of our further analysis. Figure 8 shows the velocity and velocity dispersion maps for our two kinematically distinct components. We have replaced all bins where we recovered the fractional contribution of the counter-rotating component to be less than $15 \%$ within the main stellar one with our previous single kinematiccomponent fit. The results that we obtained folding the photometric decomposition information back into our kinematic decomposition also agree to a high degree with the ones where it was left as a free parameter. The mean absolute differences between the kinematics extracted with and without incorporating the photometric decomposition are $\sim 11 \mathrm{~km} \mathrm{~s}^{-1}$ in velocity and $\sim 12 \mathrm{~km} \mathrm{~s}^{-1}$ in velocity dispersion. The velocity amplitudes for both components are again comparable with the counterrotating component reaching a maximum of $\sim 125 \mathrm{~km} \mathrm{~s}^{-1}$ at a distance of $\sim 12^{\prime \prime}$. The main component has a maximum velocity amplitude of $\sim 135 \mathrm{~km} \mathrm{~s}^{-1}$ in the outskirts of the field along roughly the major photometric axis. The counter-rotating disc component has a lower velocity dispersion and a mean value of $\sim 46 \mathrm{~km} \mathrm{~s}^{-1}$ with a maximum of $\sim 75 \mathrm{~km} \mathrm{~s}^{-1}$ in the very centre and a minimum of $\sim 30 \mathrm{~km} \mathrm{~s}^{-1}$. The main component has higher velocity dispersion with a mean value of $\sim 86 \mathrm{~km} \mathrm{~s}^{-1}$.

We report an absence of ionized-gas emission in NGC 448. Although the [O $\mathrm{III}], \mathrm{H} \beta,[\mathrm{N} \mathrm{II}]$, and $\mathrm{H} \alpha$ emission lines should normally reside in the 4700-6715 $\AA$ wavelength range, none of them had a sufficient amplitude-to-noise $(A / N)$ retrieved through our GANDALF fit to surpass the detection threshold of $A / N>3$. Furthermore, neither the Katkov et al. (2016) long-slit spectrum, nor the McDermid et al. (2015) ATLAS ${ }^{3 \mathrm{D}}$ observations with SAURON showed the presence of such emission lines. The lack of gas is further enforced by the limits put on the $\mathrm{H}_{2}$ mass $\left(\log M\left(\mathrm{H}_{2}\right)<7.74\left[M_{\odot}\right]\right)$ of Young et al. (2011) based on IRAM CO $J=1-0$ and $J=2-1$ emission observations. In addition, the galaxy is not detected in the FIRST $1.4 \mathrm{GHz}$ survey, excluding the presence of strong-enough star formation, that could serve as an ionizing source (Nyland et al. 2017).
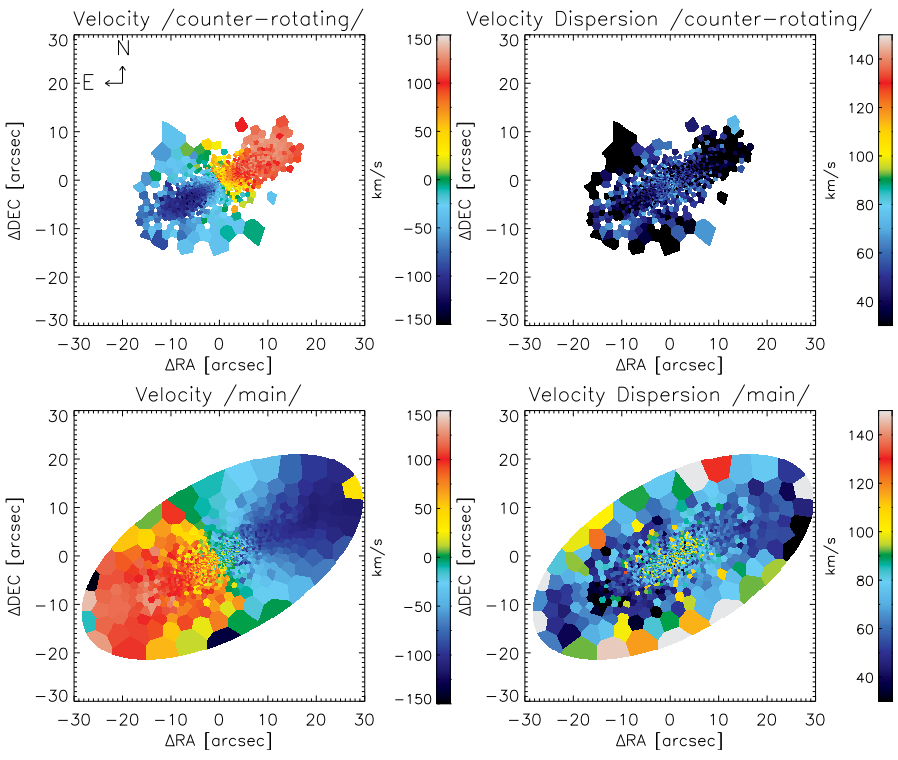

Fig. 6. The extracted kinematic maps without imposing any priors on the fractional light contribution for NGC 448. The bins are selected such that the fractional contribution of the counter-rotating component is greater than $15 \%$. Top-left panel: velocity field of the counter-rotating kinematic component. Top-right panel: velocity dispersion map for the counter-rotating component. Bottom-left panel: velocity field of the main kinematic component. Top-right panel: velocity dispersion map for the main component.

\subsubsection{NGC 4365}

We also applied the kinematic decomposition technique to NGC 4365. Figure 9 shows a spectrum of NGC 4365 as an example of our attempt at performing such a decomposition in the CaT region. Unlike the case for NGC 448, our kinematic and photometric decompositions did not return light fractions consistent with one another. Moreover, the retrieved kinematics were found to be very highly dependent on the choice of the set of initial guesses. Furthermore, the derived fractional light contribution did not display any consistent profile when left free to 

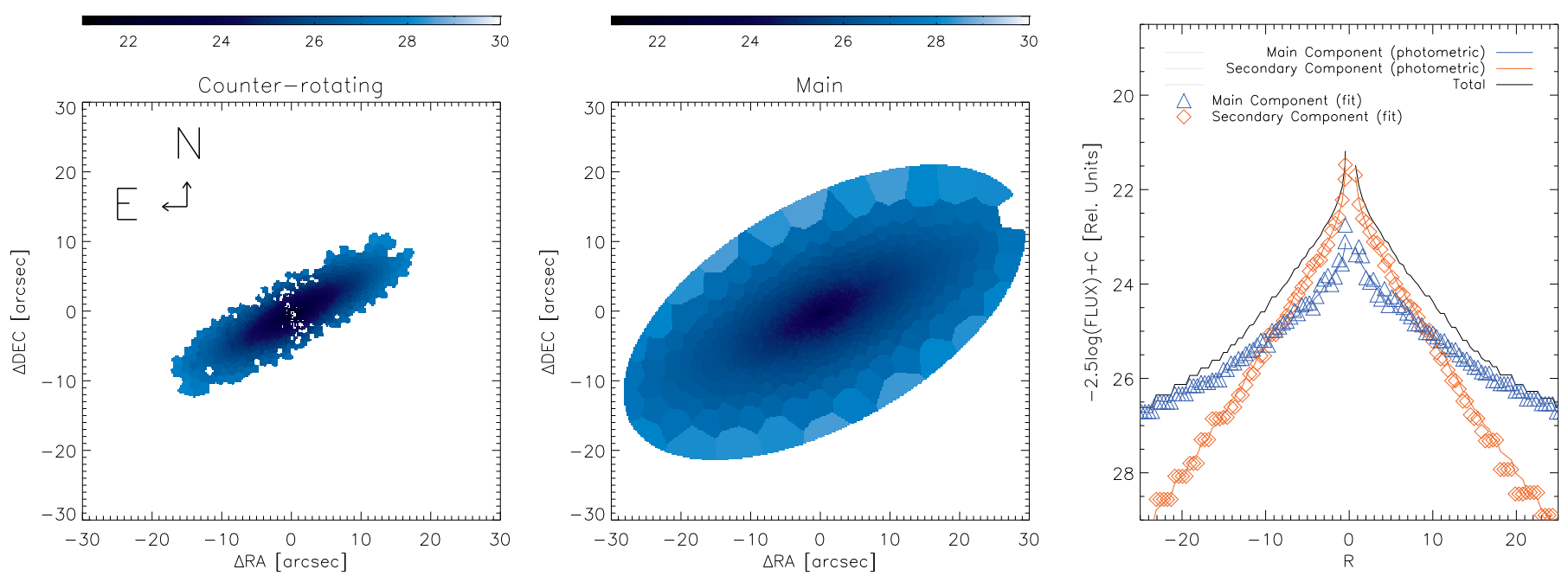

Fig. 7. Spectral flux maps averaged over the number of spaxels and profile fixed to the one recovered by the photometric decomposition for NGC 448. Left panel: reconstructed surface brightness map of the counter-rotating component. Middle panel: reconstructed surface brightness map of the main component. Right panel: radial profile for both kinematic components, taken through a pseudo slit approximately aligned to the semi-major axis. The black, orange, and blue solid lines illustrate the photometrically derived total, counter-rotating, and main component, respectively, flux contribution. The blue triangles and the orange diamonds show the flux contribution derived of the counter-rotating and main components as derived through our fitting procedure without imposing any constraints.

vary. Our photometric decomposition shows an excess of light in comparison to a pure Sérsic fit in the central regions of the galaxy (Sect. 3). The presence of this exponential-like photometric component, however, is most likely due to the structures formed from the orbital families that can be found in galaxies of triaxial intrinsic shape, as previously found for some of the non-barred slow-rotator early-type galaxies with KDCs (Krajnović et al. 2013), and not those with a truly kinematically decoupled structure. Even though we obtain a somewhat satisfactory fit to the spectra for some of the Voronoi bins using our decomposition procedure, the velocity and velocity dispersion fields we recover for the two different components are highly irregular. The highly non-symmetric LOSVD profiles in the centres of some ellipticals, such as the one present in NGC 4365, have previously been shown to be well matched by a superposition of two Gaussian forms of different width (e.g. Franx \& Illingworth 1988; Bender 1990; Rix \& White 1992). Nevertheless, this finding alone, and especially without a matching photometric signature, is not sufficient to provide enough evidence for the presence of a physically decoupled kinematic component.

\section{Line-strength indices and stellar population properties of NGC 448}

In this section we present the line-strength maps and stellar population properties of the individual kinematic components (i.e. counter-rotating disc and main stellar body) of NGC 448 where our kinematic decomposition technique successfully recovered their presence.

\subsection{Line-strength indices}

Our initial choice to extract the kinematics only in the limited CaT spectral region for NGC 448 did not prevent us from obtaining the Lick absorption line-strength indices as defined by Worthey et al. (1994). We re-fitted the NGC 448 spectra in the 4700-6715 $\AA$ wavelength range, where one finds the strongest stellar absorption lines defining the $\mathrm{H} \beta, \mathrm{Mg} b, \mathrm{Fe} 5270, \mathrm{Fe} 5335$,
Fe5406, Fe5709, Fe5782, $\mathrm{Na} \mathrm{D}, \mathrm{TiO}_{1}$, and $\mathrm{TiO}_{2}$, using the prior two-component kinematics as an initial guess for our kinematic decomposition. A small adjustment of $\pm 20 \mathrm{~km} \mathrm{~s}^{-1}$ in velocity, $\pm 20 \mathrm{~km} \mathrm{~s}^{-1}$ in velocity dispersion, and \pm 0.05 for the light fraction in the counter-rotating component was allowed in order to accommodate any expected change in the extracted kinematics. Generally, the kinematics obtained in two sufficiently distant wavelength domains (e.g. 4800-5380 $\AA$ and 8480-8750 $⿱$ ) could differ. In a varying mixture of stellar populations with different age, the fraction of young stars, outputting their light predominantly in the blue end of the spectrum, and older stars, dominant at the red end, could give rise to non-negligible $\sim 5 \mathrm{~km} \mathrm{~s}^{-1}$ differences in velocity and velocity dispersion and variation as big as $\sim 0.02$ in the $\mathrm{h} 3$ and h4 Gauss-Hermite coefficients (Arnold et al. 2014). This is especially relevant when dealing with possibly kinematically decoupled structures such as those in our investigation and also observed by Mitzkus et al. (2017). The kinematic decomposition we utilised in modelling the spectrum of our galaxies returns the best-fitting linear combination of library templates. We proceeded to extract the aforementioned line-strength indices on them. Coccato et al. (2011) carried out extensive verification by means of Monte Carlo simulations to test the ability of the best-fitting linear combination of templates to capture the underlying stellar population properties under different $\mathrm{S} / \mathrm{N}$ levels and kinematic behaviours albeit with spectra obtained using VLT/VIMOS. The systematic error levels were found to be negligible with respect to the ones driven by the $\mathrm{S} / \mathrm{N}$. We have no reason to believe that the kinematic decomposition procedure would behave differently in our specific case of applying it to MUSE spectra. To crudely estimate the error on the measurements of the line-strength indices, we adopted a constant noise level equivalent to $\sqrt{2}$ of the resistant standard deviation ("robust sigma") of the difference between the spectra and our best-fit model and the procedure outlined by Cardiel et al. (1998). Furthermore, we also evaluated the combined $[\mathrm{MgFe}]^{\prime}=\sqrt{\mathrm{Mg} b(0.82 \cdot \mathrm{Fe} 5270+0.28 \cdot \mathrm{Fe} 5335)}$ and $\langle F e\rangle=(\mathrm{Fe} 5270+\mathrm{Fe} 5335) / 2$ indices $($ Thomas et al. 2003; Gorgas et al. 1990). Both are considered to be good proxies for the total metallicity. In particular, $[\mathrm{MgFe}]^{\prime}$ has been 

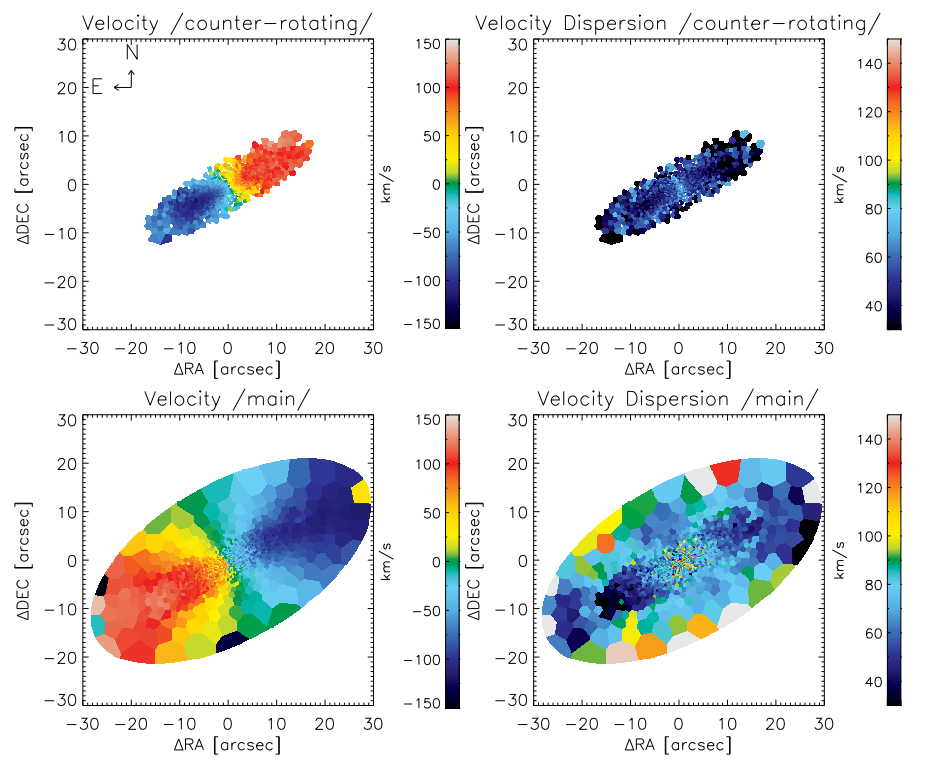

Fig. 8. The extracted kinematic maps with the fractional light contribution fixed to correspond to the photometrically derived one for NGC 448. The bins are selected such that the fractional contribution of the counter-rotating component is greater than $15 \%$. Top-left panel: velocity field of the counter-rotating kinematic component. Top-right panel: velocity dispersion map for the counter-rotating component. Bottom-left panel: velocity field of the main kinematic component. Top-right panel: velocity dispersion map for the main component.

demonstrated to be only weakly dependent on the alphaelement-to-iron abundance ratio $(\alpha / \mathrm{Fe})$, and the ratio of the $\mathrm{Mg} b$ to $\langle\mathrm{Fe}\rangle$ for old stellar populations is found to correlate reliably with the total $\alpha / \mathrm{Fe}$ enhancement (Thomas et al. 2003).

Figure 10 shows the strength of these indices for the bins where the relative light fraction of the counter-rotating component is higher than $15 \%$ and both components have velocity shifts relative to the galactic systemic one greater than $30 \mathrm{~km} \mathrm{~s}^{-1}$ with the corresponding Thomas et al. (2011) SSP model over-imposed. Within the mean errors the stellar population properties of both kinematic components show discernible differences. The strength of $\mathrm{H} \beta$ for both components is comparable, however, the two kinematic components show a noteworthy difference in their $[\mathrm{MgFe}]^{\prime}$ strengths. The two components are also offset with respect to each other in $\langle\mathrm{Fe}\rangle$ versus $\mathrm{Mg} b$ space with the counter-rotating component having higher values that cover as large a range as seen in the main stellar one, suggestive of a distinction between the $\alpha / \mathrm{Fe}$ abundance ratios of the two kinematic components.

These observations are further strengthened by inspecting the 2D maps presented in Fig. 11 for some selected line-strength indices of the counter-rotating and main components, respectively. In these maps, regions where the counter-rotating component is not reliably detected by our previously defined criteria (i.e. its light fraction $<0.15$ ) are shown in the map for the main component, adopting their values measured on the outcome of our single-component fitting procedure. The fact that we observe a smooth transition even after such a combining procedure further validates the basis for our separation.

The right panels of Fig. 11 show the profiles of the median values obtained by considering the bins contained within an elliptical aperture defined by performing a fit with the iraf task ellipse to the reconstructed white image. The velocity amplitude of the kinematic components is taken to be $>30 \mathrm{~km} \mathrm{~s}^{-1}$ as a way to safeguard against any bins where the kinematic decomposition might not have fully converged. The error bars are eval-

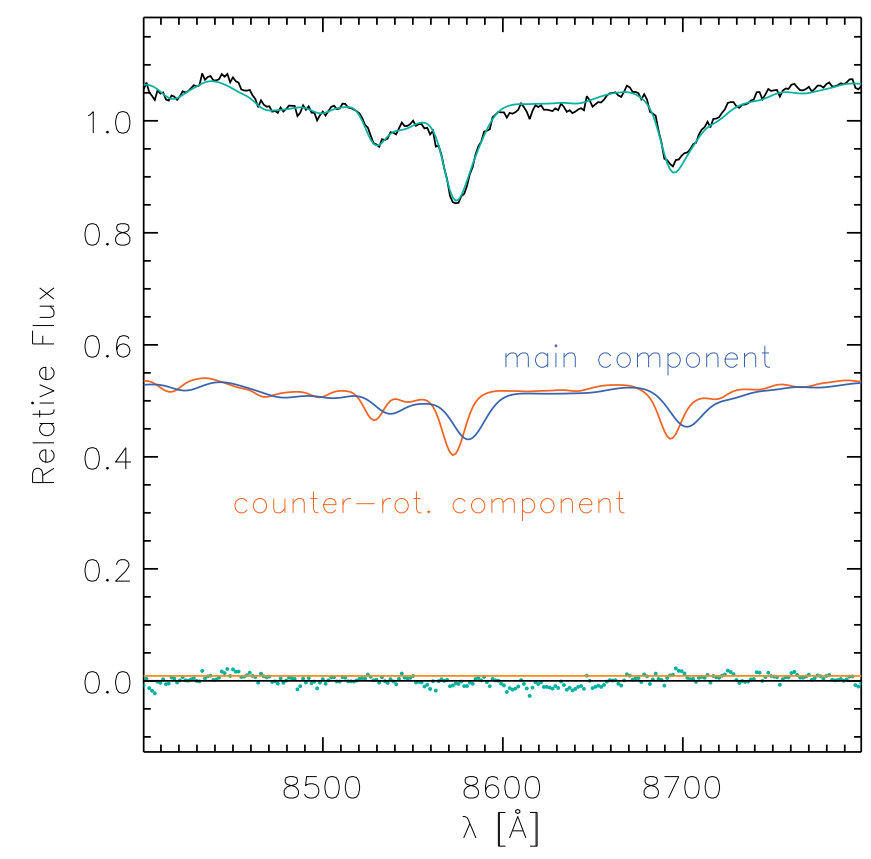

Fig. 9. Fit to the CaT spectral region of NGC 4365 for a bin approximately 10.5 arcsec away from the field center in the region where we expect a large velocity difference between the two suspected kinematic components.

uated by computing the resistant standard deviation within the apertures. At a first glance, both Figs. 10 and 11 show that the stellar-population properties of the two kinematic components are indeed different. Moreover, they both show evident radial gradients in $[\mathrm{MgFe}]^{\prime}, \mathrm{Mg} b /\langle\mathrm{Fe}\rangle$, and $\mathrm{Na} \mathrm{D}$. The counterrotating component has a lower $\mathrm{Mg} b /\langle\mathrm{Fe}\rangle$ ratio indicating that its stellar population is less enriched in $\alpha$-elements. Its metallicity as traced by $[\mathrm{MgFe}]^{\prime}$ is also systematically higher than that of the counter-rotating component. The bottom row in Fig. 11 presents the map and median radial profile for the $\alpha / \mathrm{Fe}$ insensitive $\mathrm{Na} \mathrm{D}$. We notice that this index behaves similarly to the $[\mathrm{MgFe}]^{\prime}$ one. However, due to its higher strength within the main component we could quite clearly separate the presence of a second disc and a halo component, as already suggested by the derived $[\mathrm{MgFe}]^{\prime}$ and expected ubiquitously in S0 galaxies (Guérou et al. 2016).

\subsection{Stellar population properties}

To translate our line-strength measurements into estimates for the stellar population age, metallicity and alpha-elements abundance for both the main and counter-rotating components, we applied the technique of Morelli et al. (2012) and Coccato et al. (2011) using the single-age models of Thomas et al. (2011) and the observed strength for the $\mathrm{H} \beta, \mathrm{Mg} b, \mathrm{Fe} 5270, \mathrm{Fe} 5335$, Fe5406, Fe5709, and Fe5782 absorption features. Figure 12 presents maps for the age, metallicity, and alpha-elements abundance of both stellar components in NGC 448 as well as radial profiles for the median value of these parameters evaluated in elliptical annuli. The main component is significantly older (median age of $\sim 7.7 \mathrm{Gyr}$ ) in the very central region (up to $\sim 3^{\prime \prime}$ along the major axis) and more metal enriched $([\mathrm{Z} / \mathrm{H}]$ of $\sim 0.06)$ in comparison to other regions. With this distinction, the ages of the two kinematically distinct components are comparable to each other further away from the centre. The counter-rotating component is very metal rich (median $[\mathrm{Z} / \mathrm{H}] \sim 0.15)$ and has a 
steeper gradient with values as large as $\sim 0.4$, falling to as low as $\sim 0$ in its outer parts. On the other hand, over the extent of the counter-rotating component the main component has a median metallicity of $\sim-0.05$. The alpha-enhancement of both components is observed to anti-correlate with metallicity; for the main component it ranges from $\sim 0.05$ in the central region to $\sim 0.1$ outwards. The counter-rotating component shows a hint of a reverse $\alpha$-element abundance gradient. The values are very close to Solar in the centre and rise to match the ones observed for the main component further away.

Overall, our stellar-population measurements for the two kinematic components of NGC 448 do not compare very well with the results of Katkov et al. (2016). Along the major axis, the stellar metallicity and age derived by Katkov et al. (2016) are systematically lower and higher, respectively, than our own values, for both the main and counter-rotating components. It is difficult to comment on these differences, since the analysis of Katkov et al. (2016) is based on different stellar-population models and analysis techniques from ours, namely the NBURST spectral-fitting technique of Chilingarian et al. (2007a,b) with the PEGASE.HR SSP models of Le Borgne et al. (2004). Furthermore, Katkov et al. (2016) derive only stellar ages and metallicities whereas we further explore the role of alpha-element abundances.

On the other hand, McDermid et al. (2015) use a similar modelling approach to ours, based on line-strength measurements, but only consider the overall galaxy spectrum inside circular apertures without attempting to characterise the two stellar populations of NGC 448. Nonetheless, the McDermid et al. (2015) measurements and results can serve as a test for our modelling approach. Indeed, when applying our technique with the measured strength for the $\mathrm{H} \beta, \mathrm{Mg} b, \mathrm{Fe} 5270$, and Fe5335 indices that McDermid et al. (2015) measure inside one effective radius $\left(11^{\prime \prime}\right)$ and using the Thomas et al. (2011) models, we find stellar age, metallicity, and alpha-element abundance values that are consistent with what we find in the same region with our own line-strength measurements. In the same region McDermid et al. (2015) finds older stellar ages when using the Schiavon (2007) models, suggesting that the choice of stellar-population models lies at the heart of this discrepancy.

\section{Discussion and conclusions}

The true nature of the kinematically distinct components, such as the ones observed in our two galaxies, is best studied using IFU spectroscopy, combining both photometric and kinematic information. Nevertheless, it is intrinsically difficult to reconstruct the formation mechanism for the retrograde stellar populations observed in these two early-type galaxies.

\subsection{NGC 448}

Counter-rotating kinematic components can generally originate from processes that are either external or internal to the host galaxy. The dominant formation channel for the assembly of stellar counter-rotating kinematic components is most often attributed to the accretion or reprocessing of gas and subsequent in situ star formation (Corsini et al. 2014).

On the one hand, fresh gas could be captured through a gasrich minor merger. External gas can be acquired from the companion in a retrograde fashion and is of considerate quantity (i.e. more than what is already present within the more massive galaxy) it does not become completely dissipated and swept up by pre-existing gas within the more massive host galaxy and a subsequent star-formation episode results in a population of retrograde stars. This was recently illustrated through the aid of extensive merger numerical simulations by Bassett et al. (2017) and previously addressed by Thakar \& Ryden (1996, 1998). They demonstrate how similar gas-rich minor mergers are likely to result in an S0 galaxy or did not change the morphological properties of an S0 progenitor. Usually, the resultant post-merger products of such simulations contain some appreciable gas reservoir. However, overall NGC 448 is surprisingly devoid of gas, contrary to other observed galaxies harbouring counter-rotating stellar discs. We confirm the absence of ionised-gas emission in our IFU spectra, as previously reported by Katkov et al. (2016) in their long-slit spectrum. This indicates that there is no ongoing substantial residual star formation. With these considerations, we cannot unambiguously use the coexistence of a gas reservoir and a counter-rotating disc as evidence that indeed gas accretion and subsequent in situ star-formation formed the truly kinematically decoupled structure. One possible way to reconcile such a formation scenario with our observations is to postulate that the gas has been fully consumed and processed to stars. As a consequence, the resulting recent star-formation episode should have left a sub-population of young stars predominately belonging to the counter-rotating disc. However, we do not detect any significant difference in the ages of the stellar populations of either the main galaxy body or the decoupled kinematic subcomponent. In this case, the flat age radial profile and very sharp negative metallicity gradient could be the result of a rapid outside-in formation. Still, using the recovered alpha-element abundance and Eq.(4) from Thomas et al. (2005) implies that the counterrotating disc stars formed through an extended period of star formation that lasted at least $10 \mathrm{Gyr}$. Therefore, the observed low $\alpha / \mathrm{Fe}$ values in combination with the high metallicity similar to the main galactic stellar body age of the counter-rotating component are difficult to reconcile in a purely closed-box system. The low $\alpha / \mathrm{Fe}$ values could instead be present due to accreted gas material that had already been iron enriched to lower down the $\alpha / \mathrm{Fe}$ ratio. Alternatively, star formation could have proceeded in both of the kinematic components (disc and main stellar body) generating a noticeably large ( $\geqslant 1 \%$ in mass) and young $(t \leqslant 2.5 \mathrm{Gyr})$ stellar sub-population. This in turn would impact our line-strength luminosity-weighted age estimates and metallicities, significantly shifting the age of the main stellar body towards smaller age estimates and the metallicity of the kinematically decoupled disc towards that of its oldest stellar subpopulation (e.g. Serra \& Trager 2007; Sánchez-Blázquez et al. 2014). A more thorough analysis of the stellar population properties, allowing for multiple simple stellar populations instead of just one as in this work, and using higher-resolution spectra, could suffice to either confirm or rule out such a speculation. As an alternative to a gas-rich merger, Thakar \& Ryden $(1996,1998)$ studied the possibility that the gas can fall in from the environment surrounding the galaxy through either a short or prolonged period of accretion. These numerical simulations produced counter-rotating discs, albeit most often resulting in profiles of a non-exponential nature, such as the one we recover for the counter-rotating component in NGC 448. Another possibility is that the galaxy was originally formed through filamentary gas accretion from two distinct cosmological filaments, in turn forming the two distinct kinematic structures under a special spacial configuration. Algorry et al. (2014) studied this process in a cosmological simulation of the formation of a disc galaxy. They concluded that a natural consequence of such a formation scenario would be a discriminable age difference in the two distinct kinematic components. 

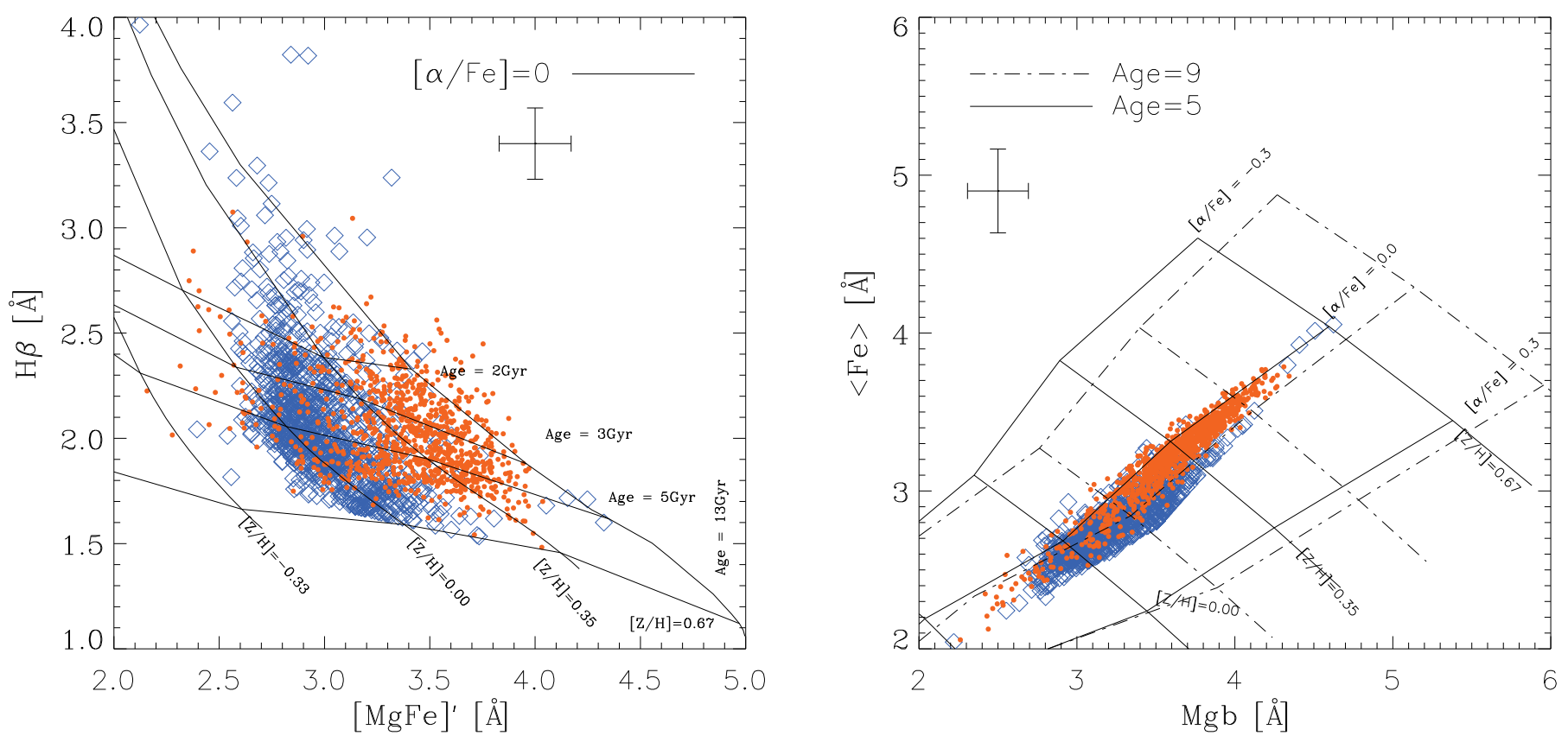

Fig. 10. Equivalent width of the composite Lick indices for NGC 448 for all bins where the two kinematic components have velocity amplitudes higher than $30 \mathrm{~km} \mathrm{~s}^{-1}$. The Thomas et al. (2011) model prediction is over-imposed. The blue diamonds signify the main component and the red filled circles indicate the counter-rotating one. The crosses show the mean error.

As previously pointed out, this is not found to be the case for NGC 448.

A further puzzle results from the likely interaction with a suspected companion galaxy (LEDA 212690). A tidal stream was observed by Duc et al. (2015) and also pointed out by Katkov et al. (2016). They note that the galaxy is in tidal interaction with a morphologically disturbed companion. From the colour difference available as part of the Duc et al. (2015) survey, we can only speculate that the age of the stars constituting the tidal tail is similar to that of the stellar population of NGC 448. If the two galaxies are interacting, then the redshift of the suspected companion LEDA 212690 was possibly wrongfully inferred as $\sim 0.074$ as part of the CAIRNS survey (Rines et al. 2003). Another more exotic possibility is that the counterrotating disc was assembled through the direct accretion of stars from a companion. It is however extremely unlikely that such a formation scenario would reproduce the observed metalicity gradient for the counter-rotating disc. There is no strong physical reason for the stars to be accreted preferentially in a spatial configuration that would produce a counter-rotating disc with higher metallicity than that of the main galaxy body and with a strong gradient. Further observations of LEDA 212690 would unequivocally rule out some of the previously mentioned external-formation scenarios for the counter-rotating disc.

As opposed to the external acquisition of gas and a subsequent in situ star formation, the counter-rotating embedded stellar disc could be the product of an internal formation mechanism. The internal separatrix formation scenario (Evans $\&$ Collett 1994) would be very unlikely in the case of NGC 448. Even though the two components have very similar stellar population ages, the counter-rotating disc is less extended than the main galaxy body, contrary to the predictions of such a formation mechanism. Furthermore, the chemical properties of the two stellar populations are quite different in opposition whereas the stars forming the counter-rotating disc should share the same star-formation history as the galaxy. Kantharia (2016) argued that counter-rotation in general could also stem from the exchange of angular momentum in close galaxy encounters. As pointed out previously we observe a "bridge" between the two galaxies. Nevertheless, we do not see any drastic misalignment in the kinematic rotation centres of the counter-rotating embedded disc and the main galactic body and we report a difference in the chemical properties of the stellar populations of the two kinematic components. Also, the two counter-rotating structures seem to share the same axis of rotation according to the accuracy of our velocity measurements (i.e. we do not observe an obvious offset between the heliocentric velocities of the two kinematic components). As the aforementioned angular momentum exchange due to a close fly-by scenario would largely result in the two components sharing different rotation axes we conclude that it is unlikely to be the origin of the separate stellar population kinematic components observed in NGC 448 .

\subsection{NGC 4365}

Even though we retrieved a second photometric component through our photometric decomposition (see, Sect. 3) and also obtained a good spectral decomposition in some of our Voronoi bins (see, Sect. 4.3.2), we did not obtain adequate consistency in our photometric and kinematic decompositions to support the presence of two truly decoupled kinematic structures. It is well established by means of simulations that the KDCs in massive early-type ellipticals could be a product of a galaxy merger. To complement the finding of the ATLAS ${ }^{3 \mathrm{D}}$, Bois et al. (2011) produced an extensive set of such merger simulations spanning a long range of possible mass ratios (1:1 to $6: 1)$, initial conditions, and orbital parameters in order to discern the origins of the two main classes of early-type galaxies on the basis of their resultant angular momentum. They report that the majority of the slow-rotator merger products in their simulations possess a distinct KDC. Additionally, a major merger of two disc galaxies even when initially both following prograde orbits (Tsatsi et al. 2015) could also produce a KDC in the resultant elliptical 

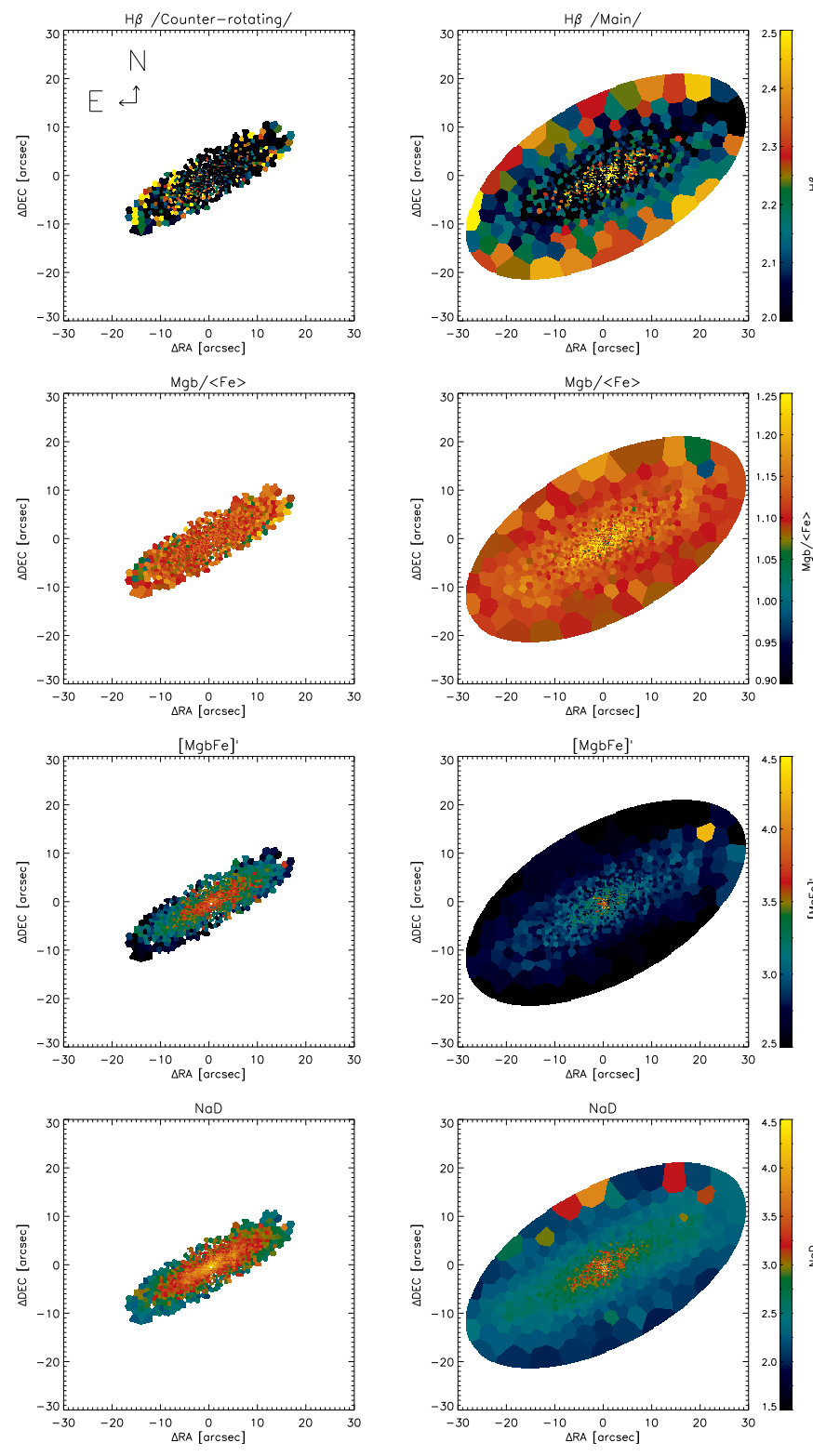
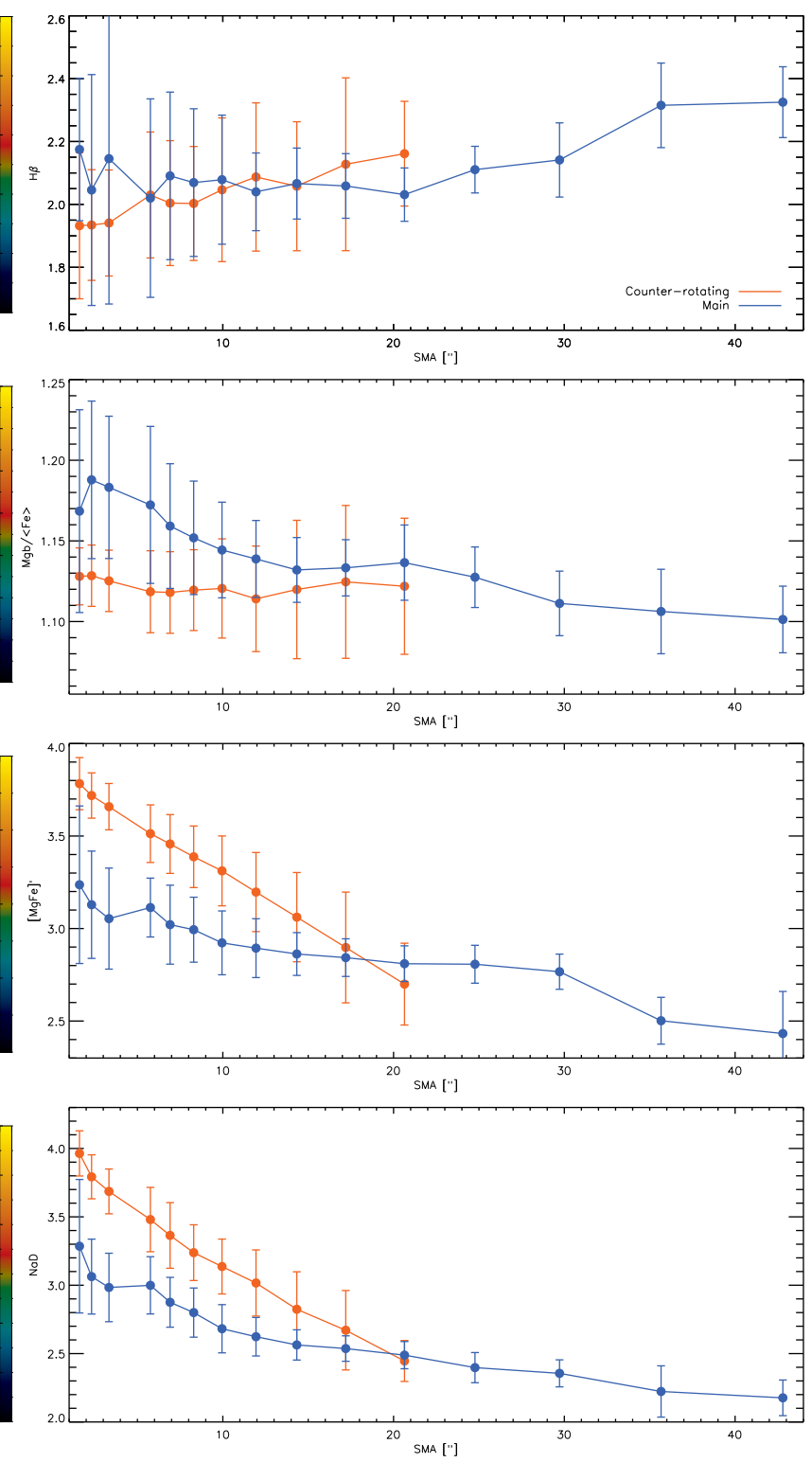

Fig. 11. Line-strength index maps and radial profiles for NGC 448. Left panels: 2D maps of the index strengths of the counter-rotating component of $\mathrm{H} \beta, \mathrm{Mg} b /\langle\mathrm{Fe}\rangle,[\mathrm{MgFe}]^{\prime}$, and $\mathrm{Na} \mathrm{D}$ from top to bottom, respectively. Middle panels: Same 2D maps of the index strengths, but for the main component. Right panels: index radial profiles taking elliptical annuli as for the counter-rotating (red) and main stellar (blue) kinematic components. The error bars represent the resistant standard deviation of the measurements within a given annulus.

galaxy merger product. However, both these KDC-formation channels would result in a noticeable age difference compared to the main stellar body. As previously found by Davies et al. (2001) and reiterated by van den Bosch et al. (2008), the ages and properties of both the KDC and the rest of the galaxy have the same magnesium-to-iron abundance ratios and virtually the same ages implying that the KDC formed at least $12 \mathrm{Gyr}$ ago or is indeed a projection effect. Furthermore, NGC 4365 does not even show symmetric velocity dispersion peaks and therefore does not resemble a typical $2 \sigma$ galaxy considered to host truly decoupled counter-rotating structures. Even in the presence of such features, for the case of NGC 5813, Krajnović et al. (2015) did not infer that the KDC arises from two counter-rotating discs. Instead through detailed modelling, as also previously done for NGC 4365 by van den Bosch et al. (2008), Krajnović et al. (2015) found that also in NGC 5813 the KDC is likely not due to two dynamically decoupled components. It was found to most plausibly be the result of the complex nature of the orbits in such massive ellipticals.

\subsection{Summary}

We investigated two early-type galaxies, NGC448 and NGC 4365, that show central kinematically distinct cores. Using the kinematic decomposition technique by Coccato et al. (2011) we separated the individual contributions of two distinct kinematic components with the aid of integral-field observations of NGC 448. This latter galaxy hosts a counter-rotating stellar kinematic structure with an angular size of at least $42^{\prime \prime}$, which is considerably more extended ( $\sim 1.5$ times) than the regions with irregular stellar kinematics. Based on the observed nearly exponential photometric profile and the kinematic information we extracted, the distinct component is very kinematically cold $\left(V_{\star} / \sigma_{\star} \gg 1\right)$, and therefore likely has a disc morphology. The 

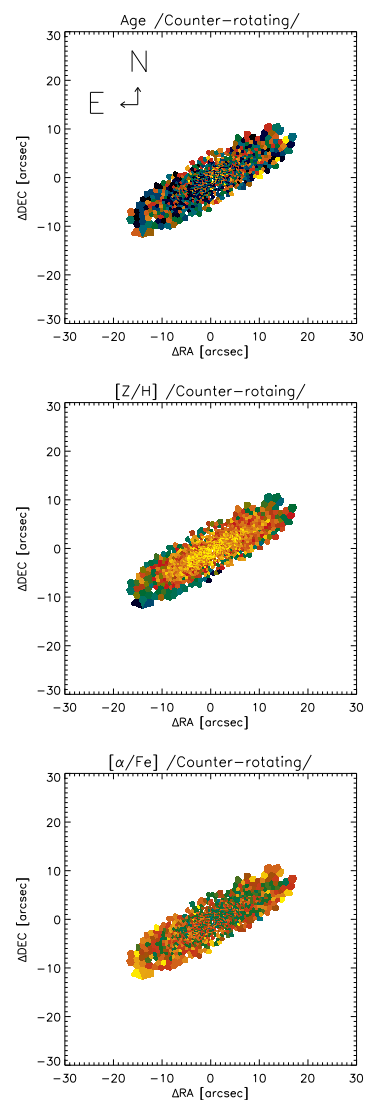
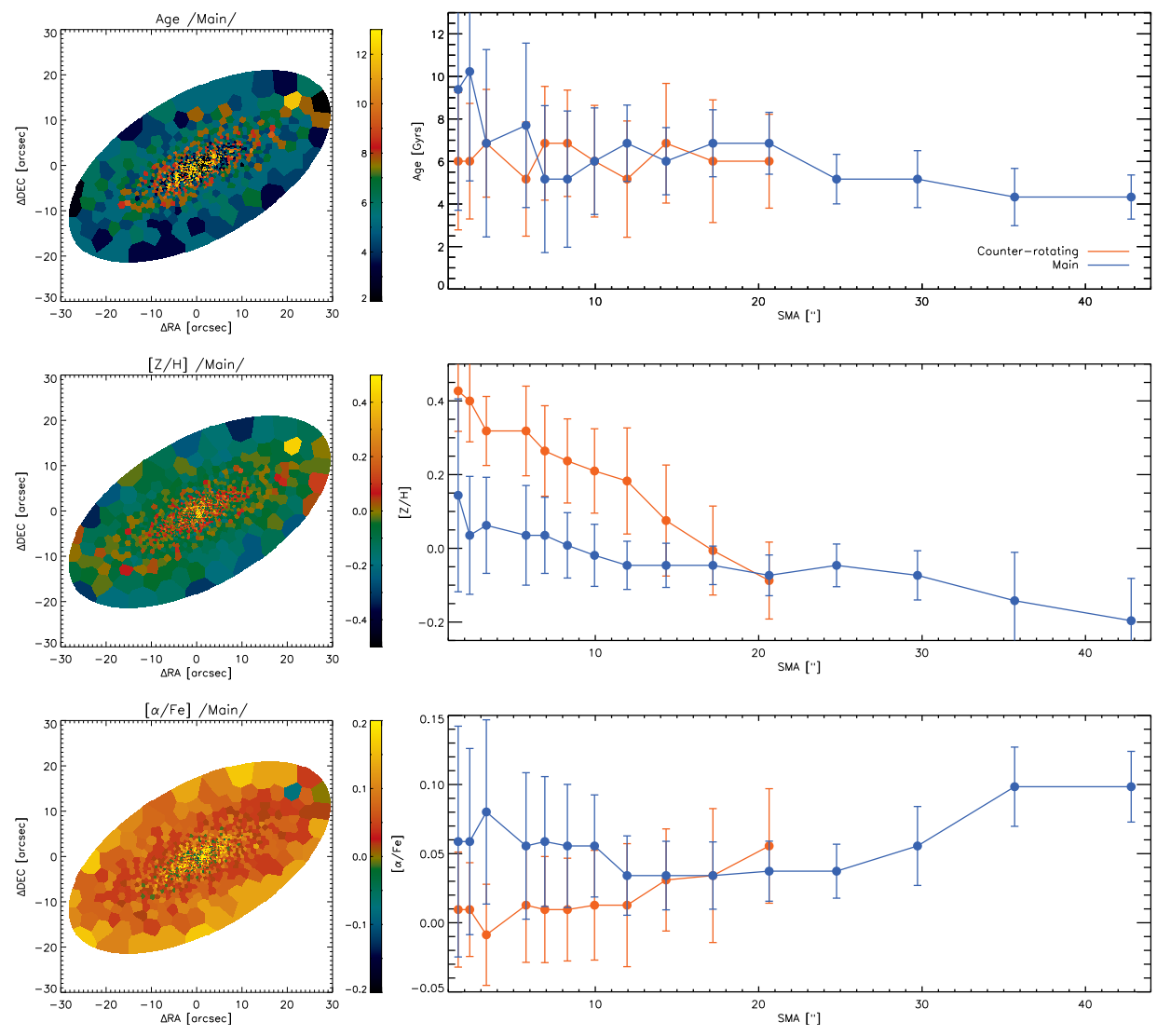

Fig. 12. Single stellar population properties for NGC 448 recovered by fitting the Thomas models. Left panel: $2 \mathrm{D}$ maps of the age, metallicity $([\mathrm{Z} / \mathrm{H}])$, and $\alpha$-element-to-iron abundance $([\alpha / \mathrm{Fe}])$ for the counter-rotating component. Middle panel: same age, metallicity, and $[\alpha / \mathrm{Fe}] \mathrm{maps}$ for the main stellar kinematic component. Right panel: radial profiles of the best-fitting ages, $[Z / H]$, and $[\alpha / \mathrm{Fe}]$ of the counter-rotating kinematic component (red) and the main one (blue). The error bars were evaluated by taking the resistant standard deviation of the values within a given annulus.

disc counter-rotates with similar velocity to that of the main stellar body. The stars of the counter-rotating disc and the main body have comparable age. However, the two distinct kinematic components have chemically different stellar populations. The stellar population of the counter-rotating disc displays a steeper metallicity gradient and is slightly less enriched in alphaelements than the one constituting the bulk of the galaxy. In contrast to all other galaxies with embedded counter-rotating discs we do not observe a significant amount of interstellar gas. Although this prohibits us from unequivocally linking the formation of the counter-rotating disc to a past gas accretion event or a "wet" merger, our analysis points to two likely formation mechanisms for the counter-rotating stellar disc. Either it formed as result of a gas-rich merger after which the gas reservoir was exhausted in a period of outside-in star formation (provided that this gas reservoir was prior iron-enriched and just acquired in retrograde orbits), or it derived from two simultaneous star-formation bursts, one of which gave rise to the counter-rotating disc, whilst the other reshaped the chemical properties of the main disc. We find no clear signature in the integral-field observations of NGC 4365 for a true kinematic decoupling. The KDC in this galaxy likely stems as previously suggested from a projection effect due to its triaxial nature.

Acknowledgements. Both BN and MS acknowledge the financial support and hospitality provided by the European Southern Observatory (ESO) through the award of an ESO studentship and visiting fellowship, respectively. EMC, EDB, LM, and AP acknowledge financial support from Padua Univer- sity through grants DOR1699945/16, DOR1715817/17, DOR1885254/18, and BIRD164402/16.

\section{References}

Algorry, D. G., Navarro, J. F., Abadi, M. G., et al. 2014, MNRAS, 437, 3596 Arnold, J. A., Romanowsky, A. J., Brodie, J. P., et al. 2014, ApJ, 791, 80 Balcells, M., \& Quinn, P. J. 1990, ApJ, 361, 381

Bassett, R., Bekki, K., Cortese, L., \& Couch, W. 2017, MNRAS, 471, 1892

Beers, T. C., Flynn, K., \& Gebhardt, K. 1990, AJ, 100, 32

Bender, R. 1988, A\&A, 202, L5

Bender, R. 1990, A\&A, 229, 441

Bertola, F., Cinzano, P., Corsini, E. M., et al. 1996, ApJ, 458, L67

Bois, M., Emsellem, E., Bournaud, F., et al. 2011, MNRAS, 416, 1654

Cappellari, M. 2016, ARA\&A, 54, 597

Cappellari, M. 2017, MNRAS, 466, 798

Cappellari, M., \& Copin, Y. 2003, MNRAS, 342, 345

Cappellari, M., \& Emsellem, E. 2004, PASP, 116, 138

Cappellari, M., Emsellem, E., Bacon, R., et al. 2007, MNRAS, 379, 418

Cappellari, M., Emsellem, E., Krajnović, D., et al. 2011, MNRAS, 413, 813

Cardiel, N., Gorgas, J., Cenarro, J., \& Gonzalez, J. J. 1998, A\&AS, 127, 597

Chilingarian, I., Prugniel, P., Sil'Chenko, O., \& Koleva, M. 2007a, in Stellar Populations as Building Blocks of Galaxies, eds. A. Vazdekis, \& R. Peletier, IAU Symp., 241, 175

Chilingarian, I. V., Prugniel, P., Sil'Chenko, O. K., \& Afanasiev, V. L. 2007b, MNRAS, 376, 1033

Coccato, L., Morelli, L., Corsini, E. M., et al. 2011, MNRAS, 412, L113

Coccato, L., Morelli, L., Pizzella, A., et al. 2013, A\&A, 549, A3

Coccato, L., Iodice, E., \& Arnaboldi, M. 2014, A\&A, 569, A83

Coccato, L., Fabricius, M., Morelli, L., et al. 2015, A\&A, 581, A65

Corsini, E. M. 2014, in Multi-Spin Galaxies, eds. E. Iodice, \& E. M. Corsini, ASP Conf. Ser., 486, 51

Crocker, A. F., Jeong, H., Komugi, S., et al. 2009, MNRAS, 393, 1255

Davies, R. L., Kuntschner, H., Emsellem, E., et al. 2001, ApJ, 548, L33 
de Zeeuw, T., \& Franx, M. 1991, ARA\&A, 29, 239

Duc, P.-A., Cuillandre, J.-C., Karabal, E., et al. 2015, MNRAS, 446, 120

Evans, N. W., \& Collett, J. L. 1994, ApJ, 420, L67

Ferrarese, L., Côté, P., Jordán, A., et al. 2006, ApJS, 164, 334

Franx, M., \& Illingworth, G. D. 1988, ApJ, 327, L55

Freudling, W., Romaniello, M., Bramich, D. M., et al. 2013, A\&A, 559, A96

Girardi, L., Bressan, A., Bertelli, G., \& Chiosi, C. 2000, A\&AS, 141, 371

Gorgas, J., Efstathiou, G., \& Aragon Salamanca, A. 1990, MNRAS, 245, 217

Guérou, A., Emsellem, E., Krajnović, D., et al. 2016, A\&A, 591, A143

Hoffman, L., Cox, T. J., Dutta, S., \& Hernquist, L. 2010, ApJ, 723, 818

Iodice, E., Coccato, L., Combes, F., et al. 2015, A\&A, 583, A48

Jedrzejewski, R. I. 1987, MNRAS, 226, 747

Johnston, E. J., Merrifield, M. R., Aragón-Salamanca, A., \& Cappellari, M. 2013, MNRAS, 428, 1296

Kantharia, N. G. 2016, ArXiv e-prints [arXiv:1606.04242]

Katkov, I. Y., Sil'chenko, O. K., \& Afanasiev, V. L. 2013, ApJ, 769, 105

Katkov, I. Y., Sil'chenko, O. K., Chilingarian, I. V., Uklein, R. I., \& Egorov, O. V. 2016, MNRAS, 461, 2068

Kormendy, J. 1984, ApJ, 287, 577

Krajnović, D., Bacon, R., Cappellari, M., et al. 2008, MNRAS, 390, 93

Krajnović, D., Emsellem, E., Cappellari, M., et al. 2011, MNRAS, 414, 2923

Krajnović, D., Alatalo, K., Blitz, L., et al. 2013, MNRAS, 432, 1768

Krajnović, D., Weilbacher, P. M., Urrutia, T., et al. 2015, MNRAS, 452, 2

Le Borgne, D., Rocca-Volmerange, B., Prugniel, P., et al. 2004, A\&A, 425, 881

Markwardt, C. B. 2009, in Astronomical Data Analysis Software and Systems XVIII, eds. D. A. Bohlender, D. Durand, \& P. Dowler, ASP Conf. Ser., 411, 251

McDermid, R. M., Emsellem, E., Shapiro, K. L., et al. 2006, MNRAS, 373, 906 McDermid, R. M., Alatalo, K., Blitz, L., et al. 2015, MNRAS, 448, 3484

Mitzkus, M., Cappellari, M., \& Walcher, C. J. 2017, MNRAS, 464, 4789

Morelli, L., Corsini, E. M., Pizzella, A., et al. 2012, MNRAS, 423, 962

Morelli, L., Pizzella, A., Coccato, L., et al. 2017, A\&A, 600, A76

Nelder, J. A., \& Mead, R. 1965, Comp. J., 7, 308

Nyland, K., Young, L. M., Wrobel, J. M., et al. 2017, MNRAS, 464, 1029

Peng, C. Y., Ho, L. C., Impey, C. D., \& Rix, H.-W. 2002, AJ, 124, 266

Pizzella, A., Corsini, E. M., Vega Beltrán, J. C., \& Bertola, F. 2004, A\&A, 424, 447

Pizzella, A., Morelli, L., Corsini, E. M., et al. 2014, A\&A, 570, A79
Puerari, I., \& Pfenniger, D. 2001, ApSS, 276, 909

Rines, K., Geller, M. J., Kurtz, M. J., \& Diaferio, A. 2003, AJ, 126, 2152

Rix, H.-W., \& White, S. D. M. 1992, MNRAS, 254, 389

Rix, H.-W., Franx, M., Fisher, D., \& Illingworth, G. 1992, ApJ, 400, L5

Rubin, V. C. 1994, AJ, 108, 456

Rubin, V. C., Graham, J. A., \& Kenney, J. D. P. 1992, ApJ, 394, L9

Salpeter, E. E. 1955, ApJ, 121, 161

Sánchez-Blázquez, P., Rosales-Ortega, F. F., Méndez-Abreu, J., et al. 2014, A\&A, 570, A6

Sarzi, M., Falcón-Barroso, J., Davies, R. L., et al. 2006, MNRAS, 366, 1151

Sarzi, M., Ledo, H. R., Coccato, L., et al. 2016, MNRAS, 457, 1804

Sarzi, M., Iodice, E., Coccato, L., et al. 2018, A\&A, 616, A121

Schiavon, R. P. 2007, ApJS, 171, 146

Schulze, F., Remus, R.-S., \& Dolag, K. 2017, Galaxies, 5, 41

Schwarzschild, M. 1979, ApJ, 232, 236

Serra, P., \& Trager, S. C. 2007, MNRAS, 374, 769

Soto, K. T., Lilly, S. J., Bacon, R., Richard, J., \& Conseil, S. 2016, MNRAS, 458, 3210

Statler, T. S. 1991, AJ, 102, 882

Tabor, M., Merrifield, M., Aragón-Salamanca, A., et al. 2017, MNRAS, 466, 2024

Thakar, A. R., \& Ryden, B. S. 1996, ApJ, 461, 55

Thakar, A. R., \& Ryden, B. S. 1998, ApJ, 506, 93

Thomas, D., Maraston, C., \& Bender, R. 2003, MNRAS, 339, 897

Thomas, D., Maraston, C., Bender, R., \& Mendes de Oliveira, C. 2005, ApJ, 621, 673

Thomas, D., Maraston, C., \& Johansson, J. 2011, MNRAS, 412, 2183

Tsatsi, A., Macciò, A. V., van de Ven, G., \& Moster, B. P. 2015, ApJ, 802, L3 van den Bosch, R. C. E., van de Ven, G., Verolme, E. K., Cappellari, M., \& de Zeeuw, P. T. 2008, MNRAS, 385, 647

van der Marel, R. P., \& Franx, M. 1993, ApJ, 407, 525

Vazdekis, A., Ricciardelli, E., Cenarro, A. J., et al. 2012, MNRAS, 424, 157

Vergani, D., Pizzella, A., Corsini, E. M., et al. 2007, A\&A, 463, 883

Weilbacher, P. M., Streicher, O., Urrutia, T., et al. 2012, in Software and Cyberinfrastructure for Astronomy II, Proc. SPIE, 8451, 84510B

Worthey, G., Faber, S. M., Gonzalez, J. J., \& Burstein, D. 1994, ApJS, 94, 687

Young, L. M., Bureau, M., Davis, T. A., et al. 2011, MNRAS, 414, 940 